\title{
Joshua Tree National Park \\ Rare Plant Habitat Suitability Analysis
}
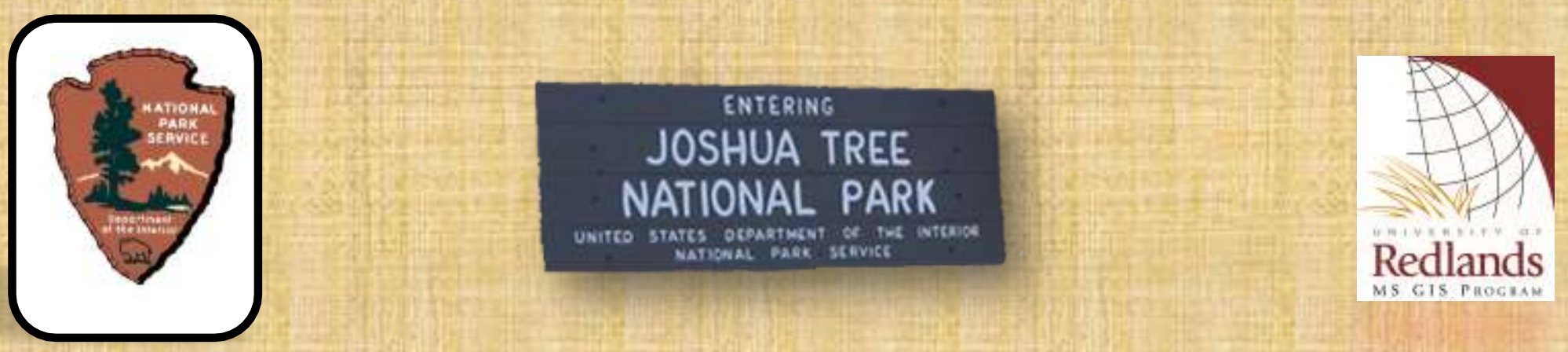


\section{Introduction}

- Client

- Important Literature

- Systems Design

- Data

- Implementation

- Results \& Analysis

- Conclusions 


\section{Client}

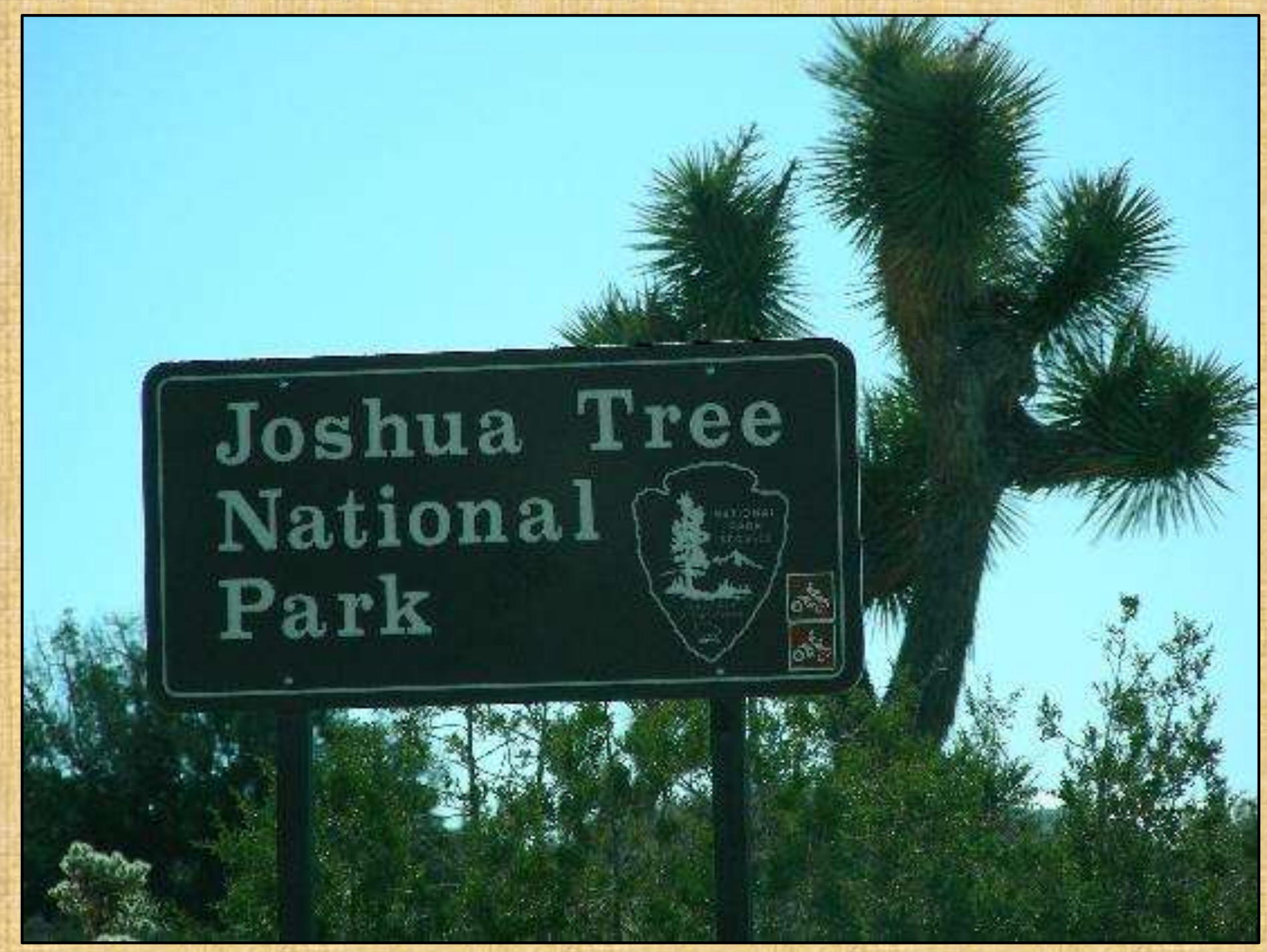




\section{Problem Statement}

JOTR needs a way to predict new rare plant habitat locations. This project aims to develop a methodology which is economical, accurate, and fast, to answer this need. This must take into consideration a lack of detailed data, and the inability to perform in-situ checks to verify findings. 


\section{Solution}

- Take JOTR data and

- Use models to

- Derive data for

- The creation of habitat index tables

- Then -

- Predict potential plant habitats with

- Weighted Overlay and Kernel Density analyses 


\section{Goals and Objectives}

1. Develop methodology

2. Create models

3. Provide simple, generalized outputs. 


\section{Pertinent Research}

- Specific habitat data unknown

- "...habitat suitability models," can be, "estimated based on expert judgments instead of empirical measurement data." (Kangas \& Store)

- Define a climactic envelope

- "The climactic envelope identifies the potential distribution range... where all environmental parameters fall within the extreme values... of observed records." (Skov) 
System Design

\section{System Requirements}

- Must have:

- Operating System

- Arc licensing level

- Thankfully:

- Client OS: Windows XP

- ArcGIS: 9.3.1 
System Design

\section{Project Requirements}

\section{- hathat?}




\section{Functional Requirements}

\section{- Create models that:}

\begin{tabular}{|l|l|}
\hline Requirement & Description \\
\hline Create Buffers & Create two buffers for each plant point (30m and 50m) \\
\hline Create points & Clip rasters to 50m buffer, create points from raster values \\
\hline Clip Points & Clip remaining points to 30m buffer \\
\hline Export Soils & $\begin{array}{l}\text { Select soils based on association with plant points, and export them } \\
\text { to a new feature class }\end{array}$ \\
\hline $\begin{array}{l}\text { ASCII } \\
\text { conversion }\end{array}$ & $\begin{array}{l}\text { Convert ASCII files to usable format, define projection, transform } \\
\text { and project. }\end{array}$ \\
\hline $\begin{array}{l}\text { Clip files to } \\
\text { JOTR }\end{array}$ & Clip the converted ASCII files to JOTR extent \\
\hline $\begin{array}{l}\text { Delete Non- } \\
\text { Near Features }\end{array}$ & $\begin{array}{l}\text { Select all features that are not equal to (<>) FIDs specified in the } \\
\text { plant's near table. }\end{array}$ \\
\hline
\end{tabular}

- Create Data 


\section{System Design}

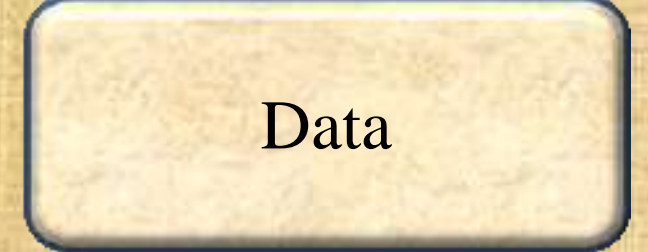

Implementation

Results

\section{Non-functional Requirements}

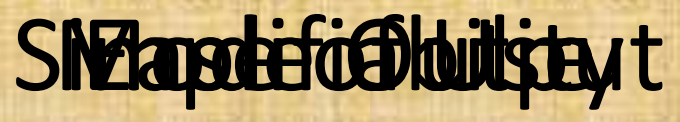

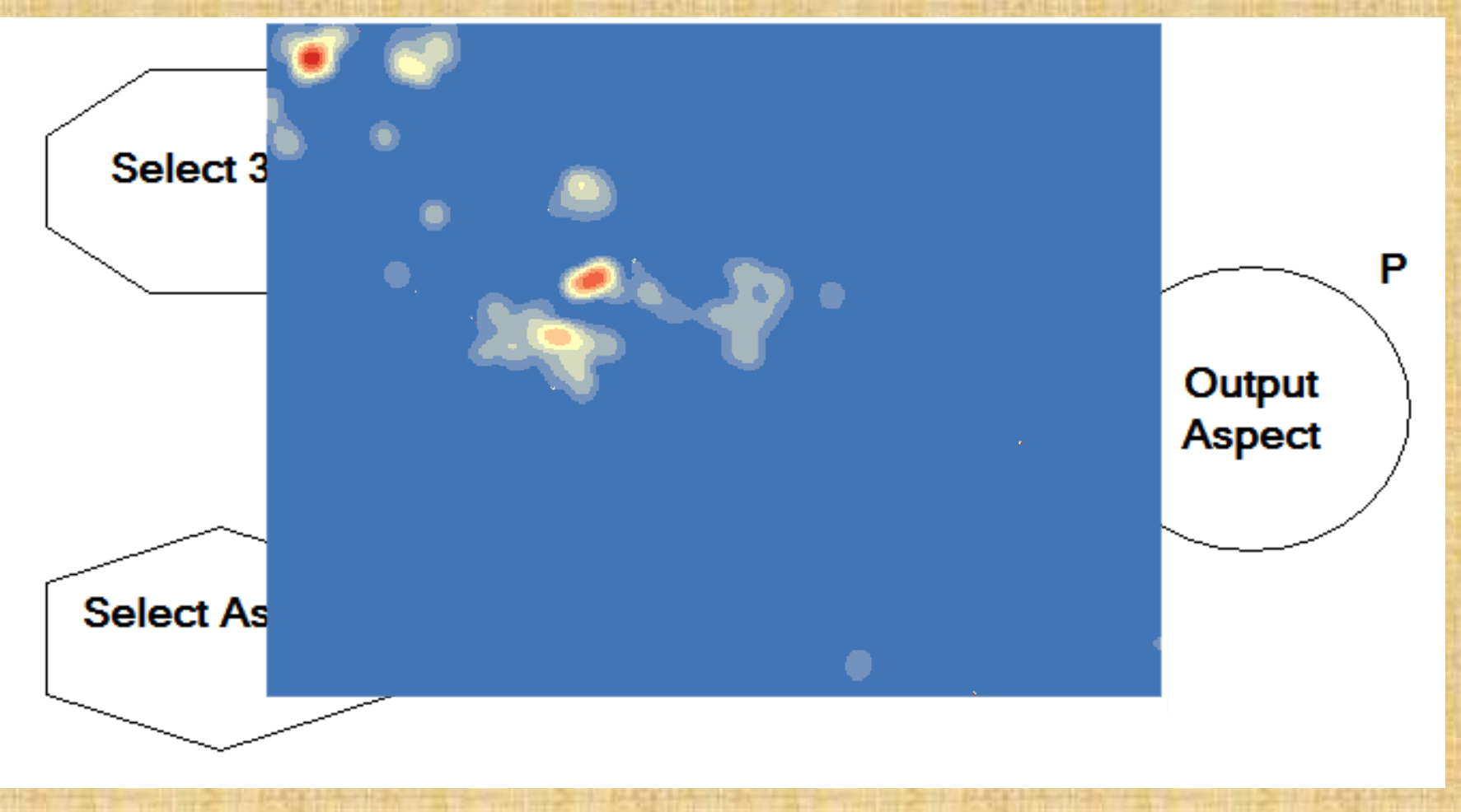




\section{System Design}

\section{Results}

\section{Database Design}

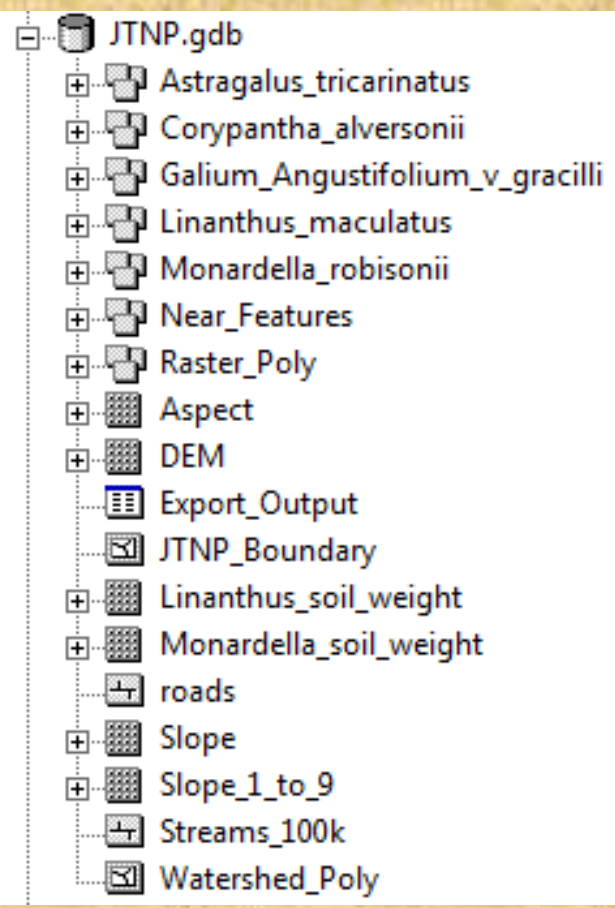
VM
W Work
-1... Astragalus.gdb
†... Corypantha.gdb
$\bigoplus$ Galium.gdb
† JTNP.gdb
Đ Linanthus.gdb
†... Monardella.gdb

Astragalus.gdb

Astragalus_tricarinatus

Astragalus_30

$\because$ Astragalus_Aspect_points

$\because$ Astragalus_DEM_points

$\because$ Astragalus_Slope_points

国 astragalus_soils_all

$\because$ Astragalus_tricarinatus

$\because$ Astragalus_tricarinatus_1

$\because$ Astragalus_weight_centroid

团 Soils_weight_poly

†.蛧 Aspect_Clip

Aspect_Reclass

芰.

†.䒽 Astragalus_weighted_overlay

+.

†.网 DEM_Reclass

†. Kernel_1500m_150M

†.网 Kernel_50m_5m

Slope_Clip

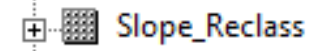


System Design

\section{Data}

\section{Data \& Data Sources}

- From Joshua Tree:

- Plants

- JOTR DEM

- Soils

- From Oregon State University:

- PRISM Temperature \& Precipitation 


\section{System Design}

\section{Conceptual Model}

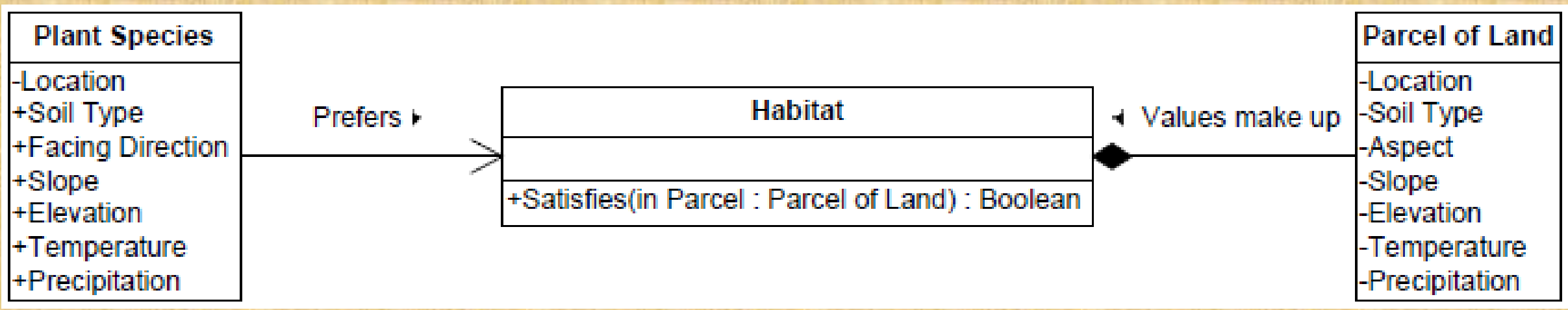




\section{System Design}

\section{Implementation}

\section{Results}

\section{Logical Model}

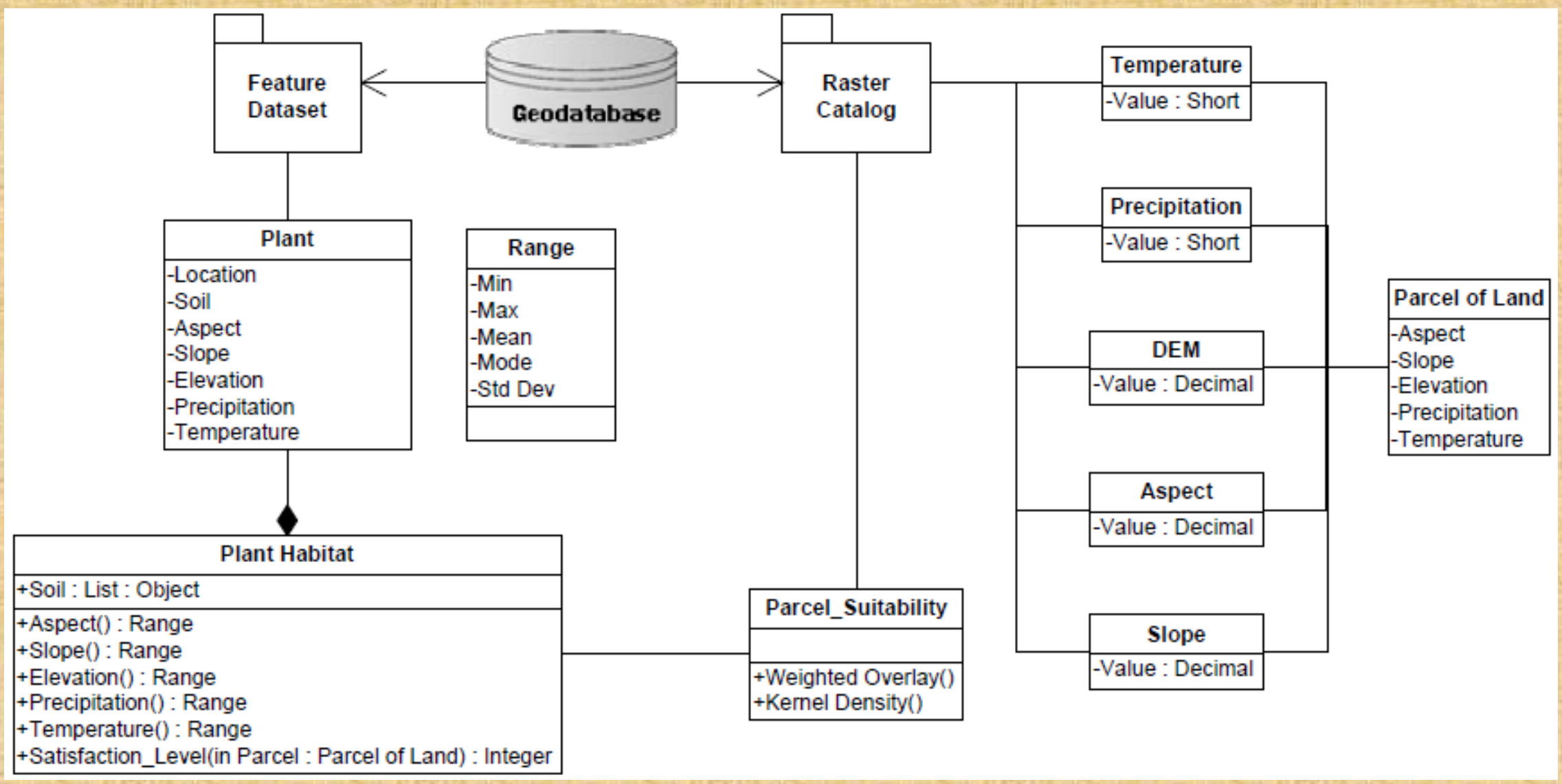




\section{Data Scrubbing \& Loading}

\begin{tabular}{|c|c|c|c|c|c|c|c|c|c|c|}
\hline & & & & & & & & & & \\
\hline 1 & OBJECTID & CNPS & SPECIES & NUM_IND & DATE_ & LOCATION & DESCRIPTI & I OBSERVEF & & $\mathrm{N}$ \\
\hline 2 & 1191 & 4.3 & Tetracocc & 1 & \#\#\#\#\#\#\# & Wilson $\mathrm{Ca}$ & In Wash at & Mike Bell, & 596093 & 3755127 \\
\hline 3 & 1192 & 4.3 & Tetracoccı & 2 & \#\#\#\#\#\#\# & southwes & growing o & TLD, B. E & & $\mathrm{N}$ \\
\hline 4 & 1193 & 4.3 & Tetracoccl & 2 & \#\#\#\#\#\#\# & southwes & growing o & TLD, B. & 596093 & 3755127 \\
\hline 5 & 1194 & 4.3 & Tetracocc & 0 & 1941 & & Riverside & & נינד & (3) \\
\hline 6 & 1195 & 4.3 & Tetracoccı & 0 & 1965 & & Riverside & & 619316 & 3728584 \\
\hline 7 & 1196 & 4.3 & Tetracoccı & 0 & 1994 & & Southern & & 619380 & 3728750 \\
\hline 8 & 1197 & 4.3 & Tetracocc & 0 & 1994 & & Sonoran D & & 611077 & 3727723 \\
\hline 9 & 1198 & 4.3 & Tetracoccl & 0 & 1998 & & Riverside & & 620255 & 3735320 \\
\hline 10 & 1199 & 4.3 & Tetracoccl & 0 & 1998 & & South slop & & & \\
\hline 11 & 1200 & 4.3 & Tetracocc & 4 & \#\#\#\#\#\#\# & Eagle Mtn & occurring & TLD, E. & 11180 & $3 / 300$ \\
\hline 12 & 1201 & 4.3 & Tetracocc & 0 & \#\#\#\#\#\#\# & Pinto Basi & Part of lar: & M. Bell & 626431 & 3746259 \\
\hline 13 & 1202 & 4.3 & Tetracoccl & 0 & \#\#\#\#\#\#\# & Pinto Basi & Part of lar & M. Bell & 622051 & 3731323 \\
\hline 14 & 1203 & 4.3 & Tetracocc & 0 & \#\#\#\#\#\#\# & Pinto Basi & Part of lar & M. Bell & 631291 & 3733386 \\
\hline 15 & 1204 & 4.3 & Tetracocc & 0 & \#\#\#\#\#\#\# & Pinto Basi & Part of lar: & M. Bell & 618708 & 3728251 \\
\hline 16 & 1205 & 4.3 & Tetracoccı & 0 & \#\#\#\#\#\#\# & Pinto Basi & Part of lar & M. Bell & & \\
\hline 17 & 1206 & 4.3 & Tetracoccl & 0 & \#\#\#\#\#\#\# & Pinto Basi & Part of lar & M. Bell & 6114 & 3738 \\
\hline 18 & 1207 & 4.3 & Tetracocc & 0 & \#\#\#\#\#\#\# & Pinto Basi & Part of lar & M. Bell & 611478 & 3738062 \\
\hline 19 & 1208 & 4.3 & Tetracoccı & 0 & \#\#\#\#\#\#\# & Pinto Basi & Part of lar & M. Bell & 611626 & 3737785 \\
\hline 20 & 1209 & 4.3 & Tetracocci & 0 & \#\#\#\#\#\#\# & Pinto Basi & Part of lar & M. Bell & 611626 & 3738137 \\
\hline 21 & 1210 & 4.3 & Tetracocc & 0 & \#\#\#\#\#\# & Pinto Basi & Part of lar & M. Bell & $0110<0$ & 3730137 \\
\hline 22 & 1211 & 4.3 & Tetracocc & 0 & \#\#\#\#\#\#\# & Pinto Basi & Part of lar & M. Bell & 611679 & 3737760 \\
\hline 23 & 1212 & 4.3 & Tetracoccl & 0 & \#\#\#\#\#\#\# & Pinto Basi & Part of lar: & M. Bell & 611879 & 3737881 \\
\hline 24 & 1213 & 4.3 & Tetracocc & 0 & \#\#\#\#\#\#\# & Pinto Basi & Part of lar & M. Bell & 612875 & 3737472 \\
\hline 25 & 1214 & 4.3 & Tetracoccı & 0 & \#\#\#\#\#\# & Pinto Basi & Part of lar & M. Bell & 613029 & 3738218 \\
\hline 26 & 1215 & 4.3 & Tetracocci $A$ & & \#\#\#\#\#\# & Lost Palm: & Populatio & T. Lado & 613154 & 3737451 \\
\hline 27 & 1216 & 4.3 & Tetracocci & & \#\#\#\#\#\# & Lost Palm: & Populatio & T. Ladc & & \\
\hline 28 & 1217 & 4.3 & Tetracocc & 0 & 2006 & & On slope i & i M. Bell & 613211 & 3737392 \\
\hline 29 & 1218 & 4.3 & Tetracocc & 5 & \#\#\#\#\#\# & Pinto Basi & On desert & M. Bell & 0204YY & 3/১UชY4 \\
\hline 30 & 1219 & 4.3 & Tetracocc & 6 & \#\#\#\#\#\#\# & Cottonwo & rocky slop & TLD, A. Mi & 610204 & 3733597 \\
\hline 31 & 1220 & 4.3 & Tetracoccl & 0 & \#\#\#\#\#\#\# & & & N. Piatrasi & 614860 & 3742622 \\
\hline 32 & 1221 & 4.3 & Tetracoccı & 0 & \#\#\#\#\#\#\# & & & N. Piatrasi & 615476 & 3742378 \\
\hline
\end{tabular}

Opuntia b $2147 \mathrm{ft}$

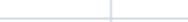

Viguieria | $2136 \mathrm{ft}$

Viguieria|2150ft

titude Longitude Associater elev $\quad$ Voucher_INotes

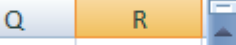

Q 


\section{System Design}

Data

\section{Data Symbolization}

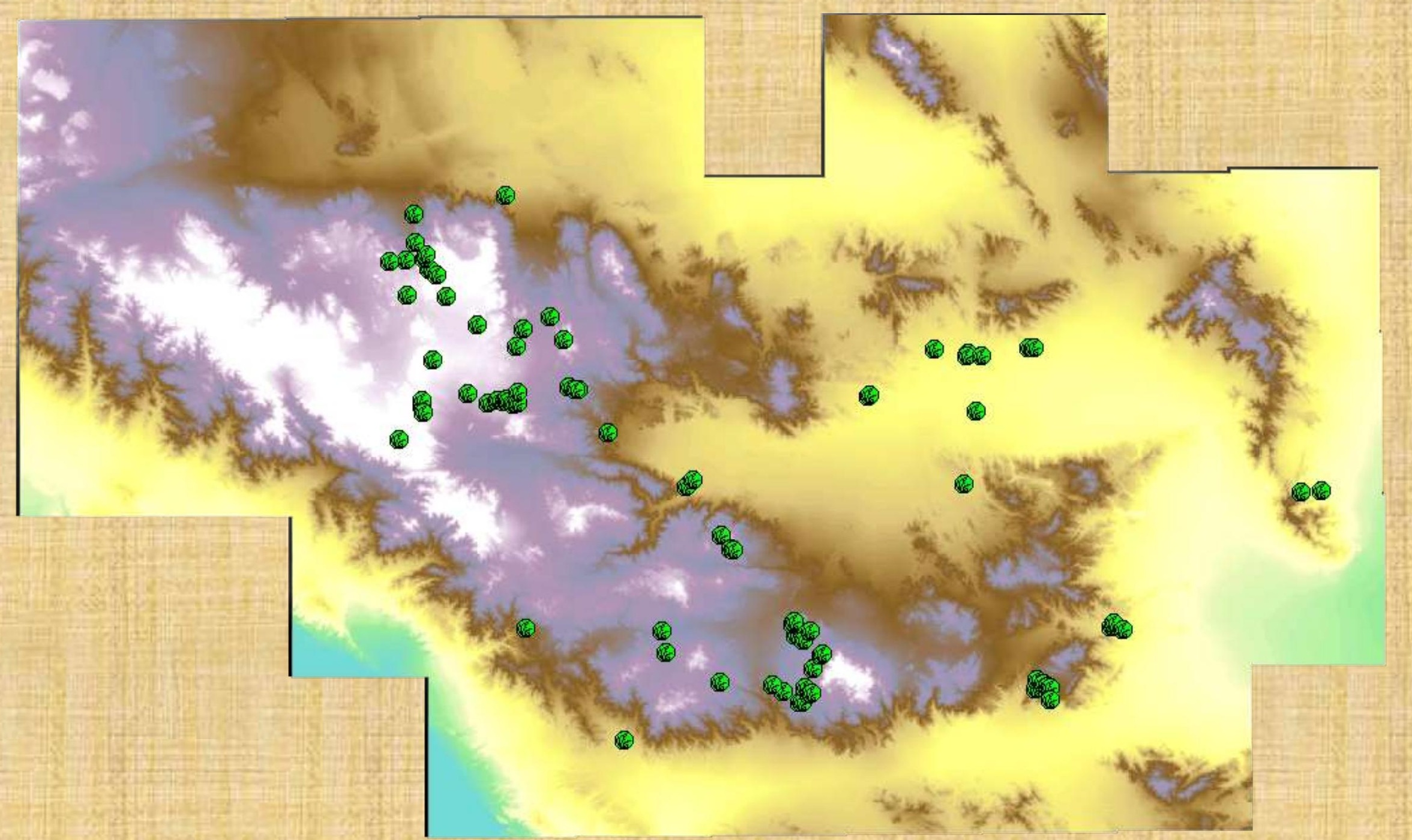




\section{System Design}

\section{Data}

Implementation

Results

\section{Data Symbolization}

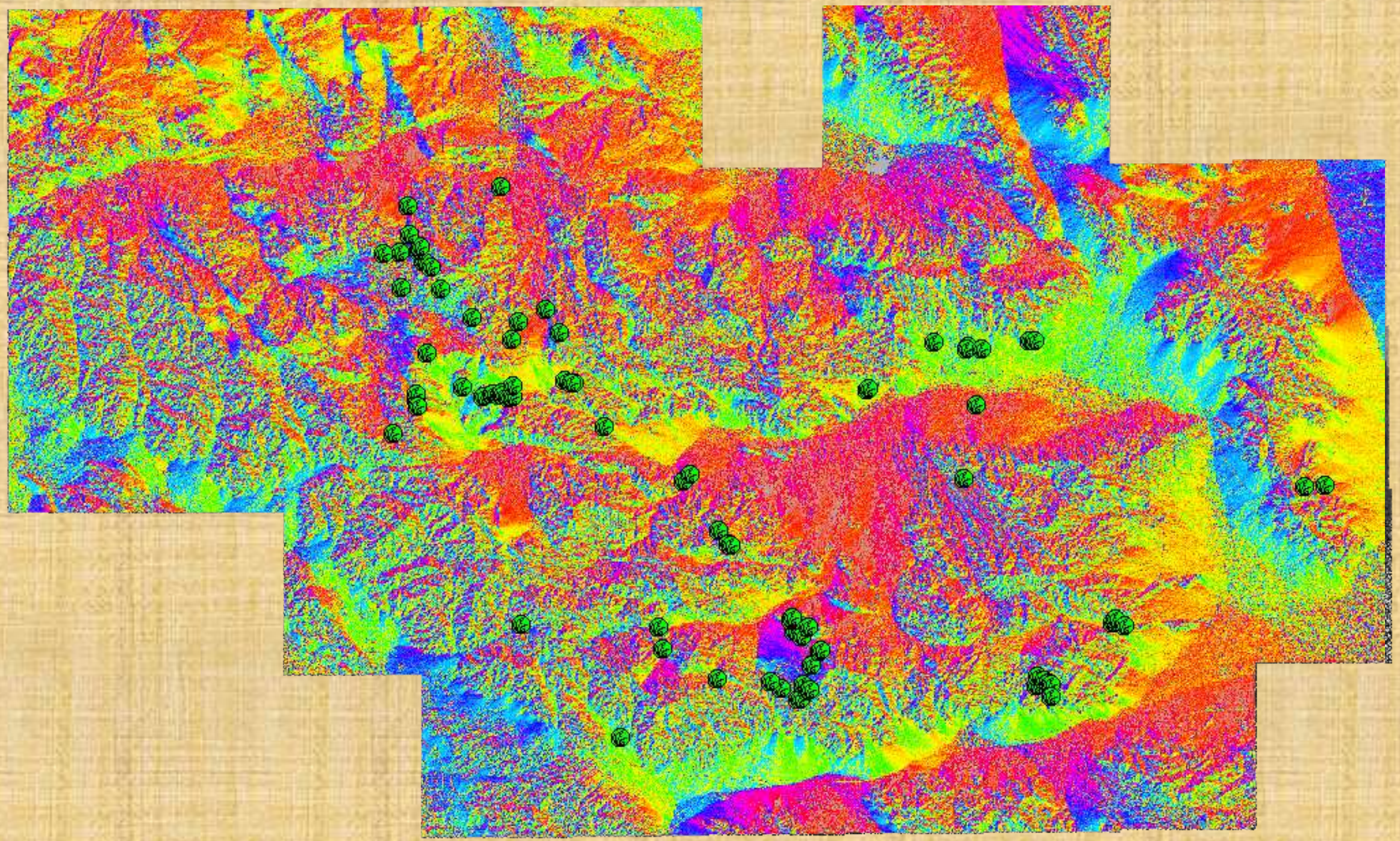




\section{System Design}

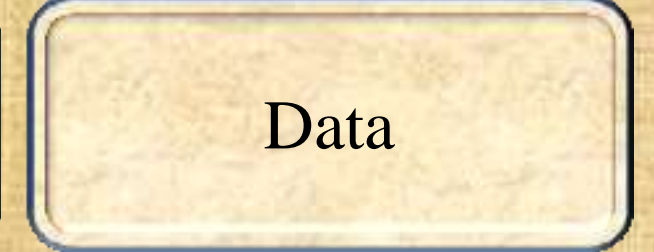

Results

\section{Data Symbolization}

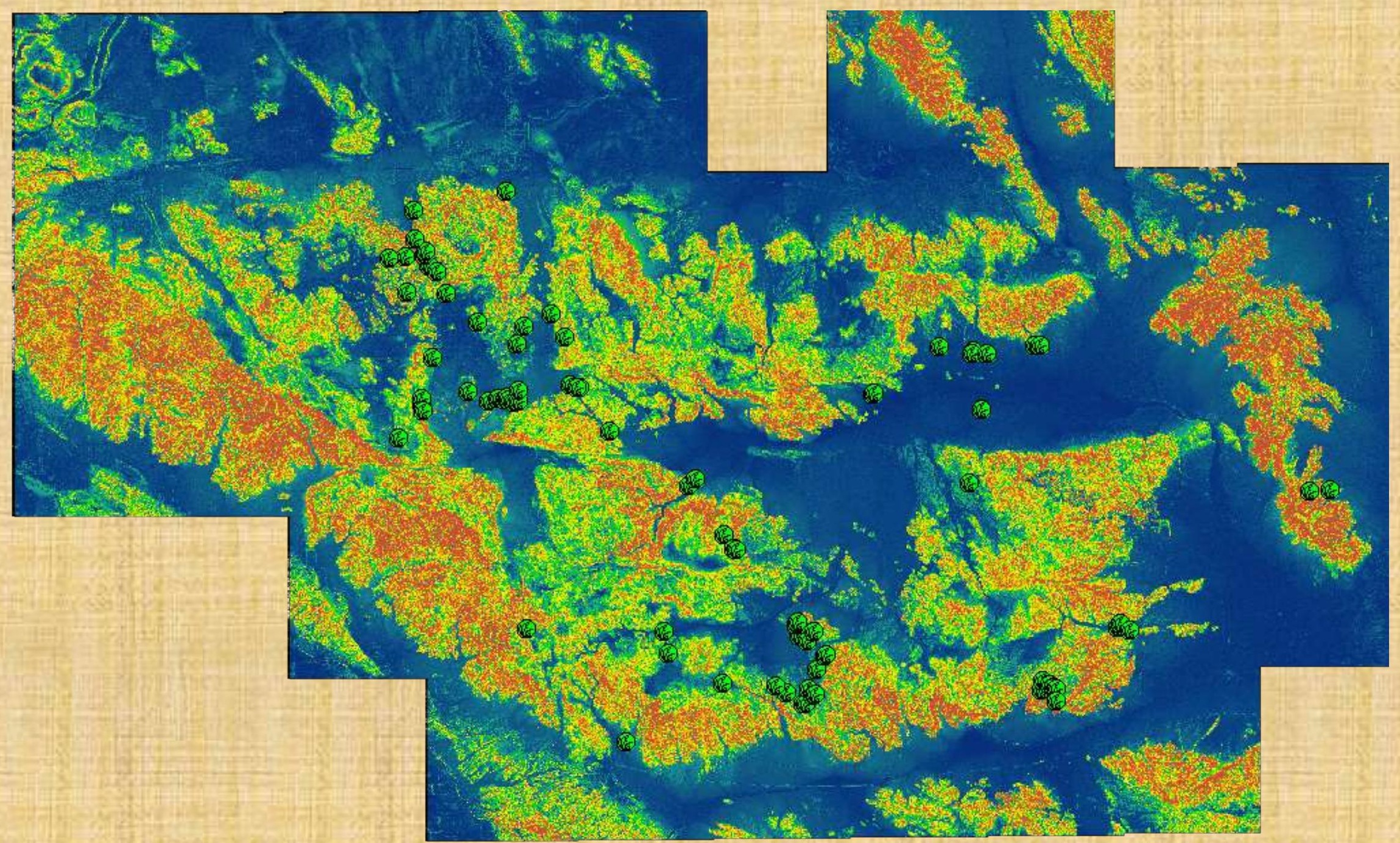


Data Symbolization

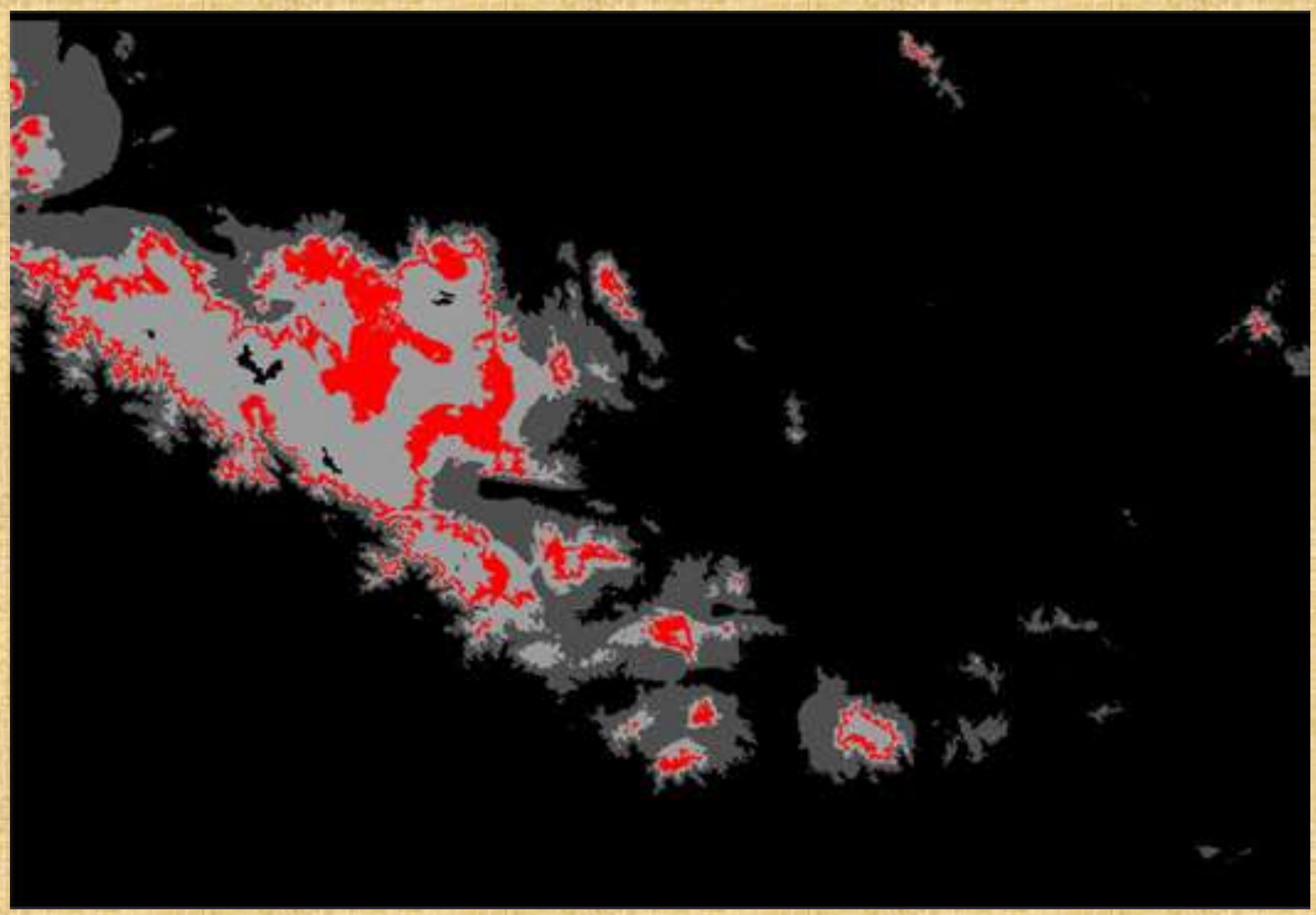




\section{Implementation}

1. Values needed to be extracted from the data

2. The data needed to be combined

$$
\text { - But - }
$$

3. Each dataset differed in overall importance

- Step 1: Preliminary visual analysis

- Step 2: Define and export values

- Step 3: Excel modification 


\section{System Design}

\section{Implementation}

\section{Results}

\section{Soil Data}

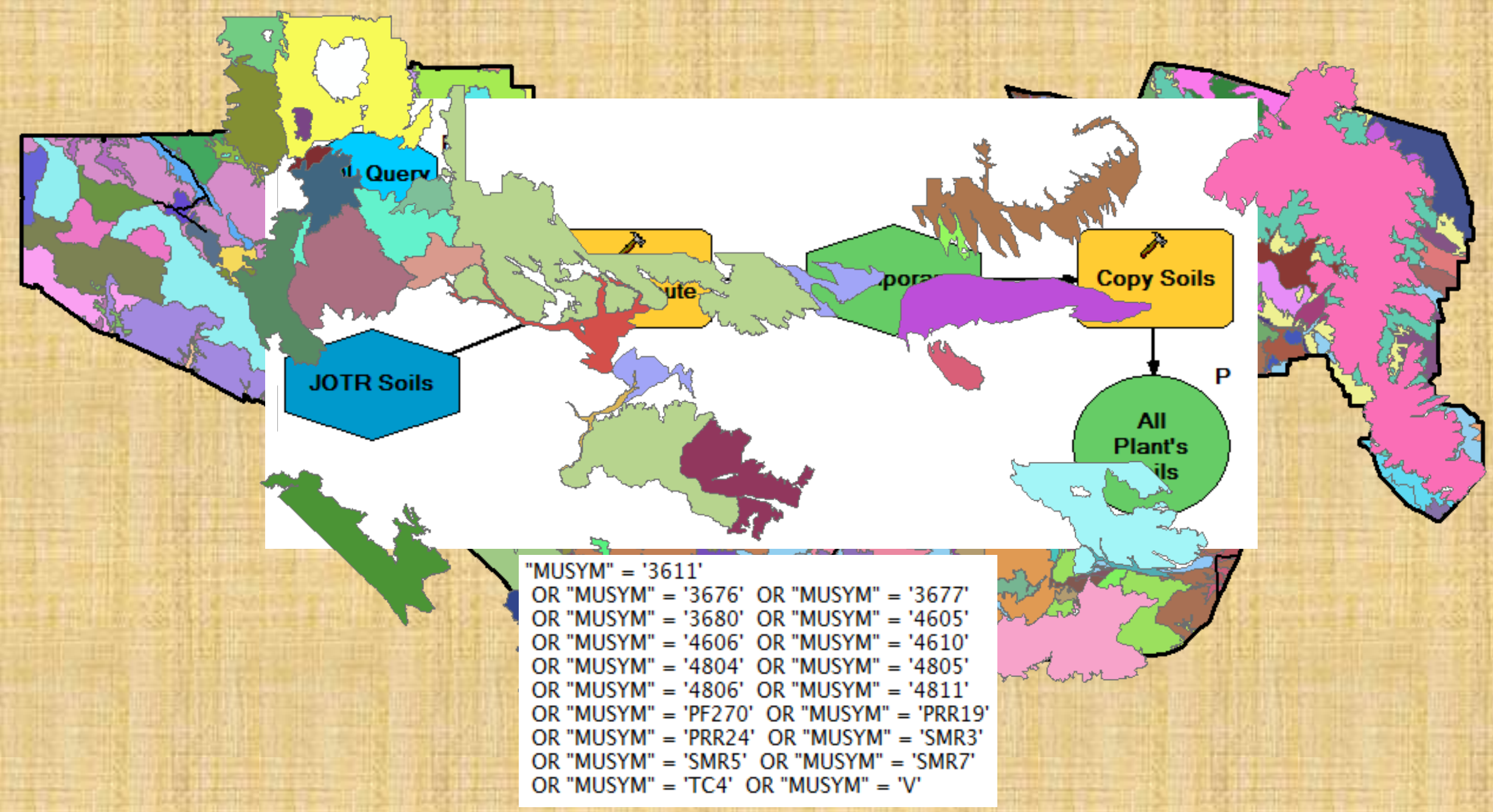




\section{System Design}

Data

Implementation

\section{Results}

\section{Data Extraction}

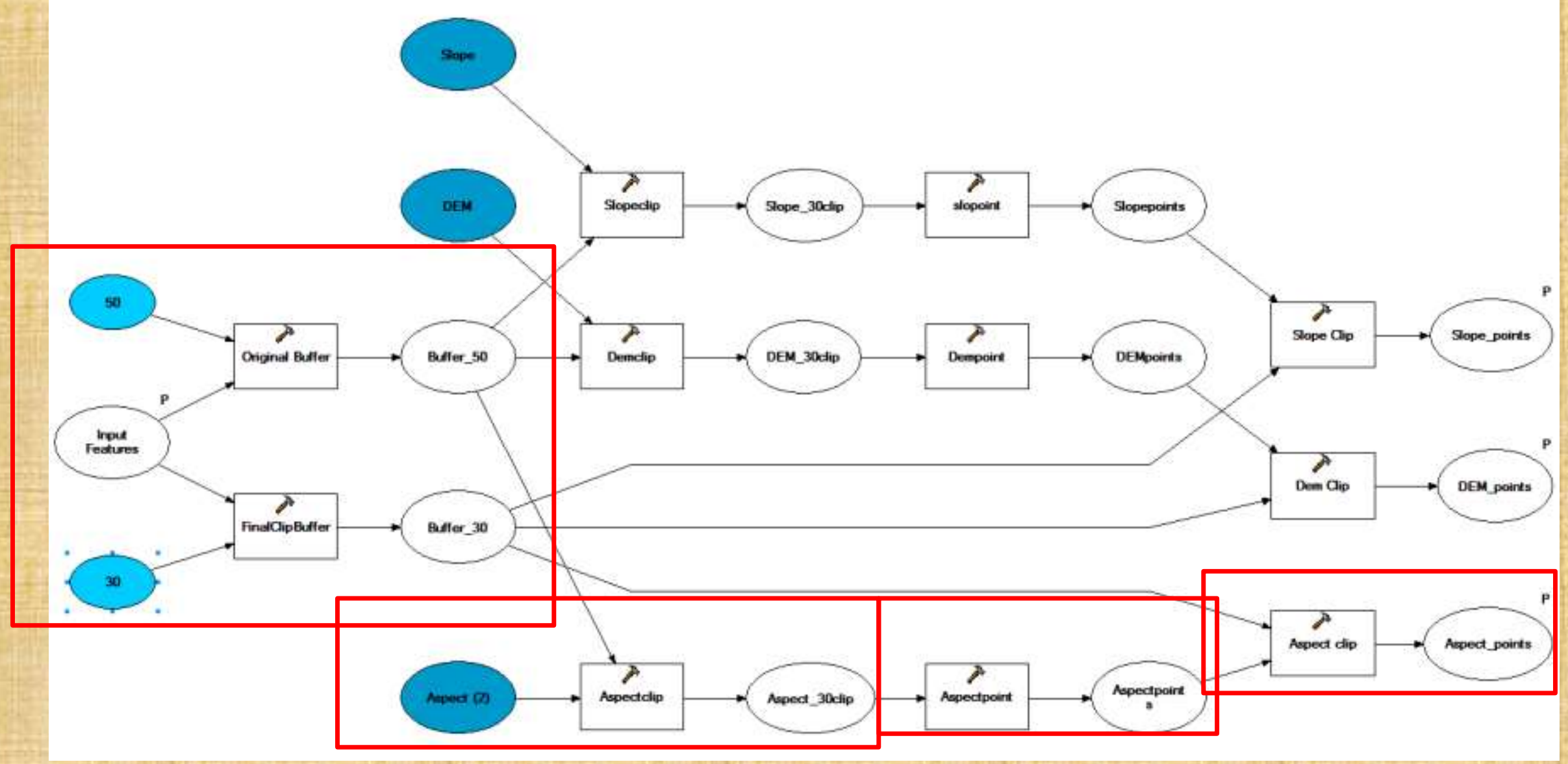




\section{System Design}

国 us_ppt_2005.11.gz

$0.99 \mathrm{MB}$

(72 us_ppt_2005.12.gz

$0.98 \mathrm{MB}$

us_ppt_2006.01.gz

az. GZ File

us_ppt_2006.02.gz

(72) GZ File

us_ppt_2006.03.gz

77. GZ File

us_ppt_2009.11.gz

[7] GZ File

us_ppt_2009.12.gz

(72) GZ File

us_ppt_2010.01.gz 77. GZ File

\section{Temperature \& Precipitation}

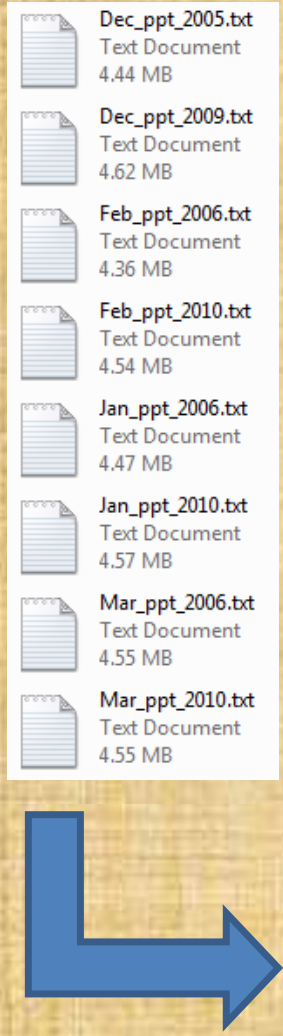

hcols 1405nrows 621xllcorner -125.020833yllcorner 24.062500cellsize 0.041667NODATA_value -9999-9999 - 9999 - 9999 - 9999 - 9999 -9999 -9999 -9999 -9999 -9999 - 9999 -9999 - 9999 - 9999 - 9999 - 9999 -9999

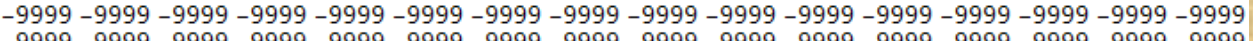
-999 - 9999 - 9999 - 9999 -9999 -9999 -9999 -9999 - 9999 -9999 -9999 -9999 -9999 -9999 -9999 -9999

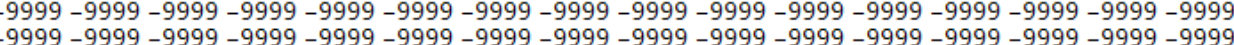
- 9999 - 9999 - 9999 - 9999 - 9999 - 9999 - 9999 - 9999 - 9999 - 9999 - 9999 - 9999 - 9999 - 9999 - 9999 - 9999 - 9999 - 9999 - 9999 - 9999 - 9999 - 9999 - 9999 - 9999 - 9999 - 9999 - 9999 - 9999 - 9999 _ 9999 - 9999 - 9999 -9999 - 9999 - 9999 - 9999 (999 (9999 פوפو وפؤ-9999 - 9999 - 9999 - 9999 - 9999 -9999 - 9999 - $9999-9999-9999-9999-9$ -9999 - 9999-9999-9999-9999-9 促 $\begin{array}{rr}-9999-9999-9999-9999-99 & -9999-9999-99 \\ -9999-9999-9999-9999-99 & -9990-9990-90\end{array}$ $-9999-9999-9999-9999-99$ $-9999-9999-9999-9990-00$ $-9999-9999-9999-999$ $-9999-9999-9999-999$ $-9999-9999-9999-999$ $-9999-9999-9999-999$ $-9999-9999-9999-999$ $-9999-9999-9999-999$ -9999 - $9999-9999-999$ -9999 - 9999 - 9999 - 9999 - 9999 -

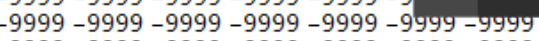

-9999 -9999 - 9999 - 9999 - 9999 - 9999 -9999 -9999 -9999 -9999 -9999 -9999 -9999 -9999 -999 


\section{System Design}

\section{Data}

\section{Temperature \& Precipitation}

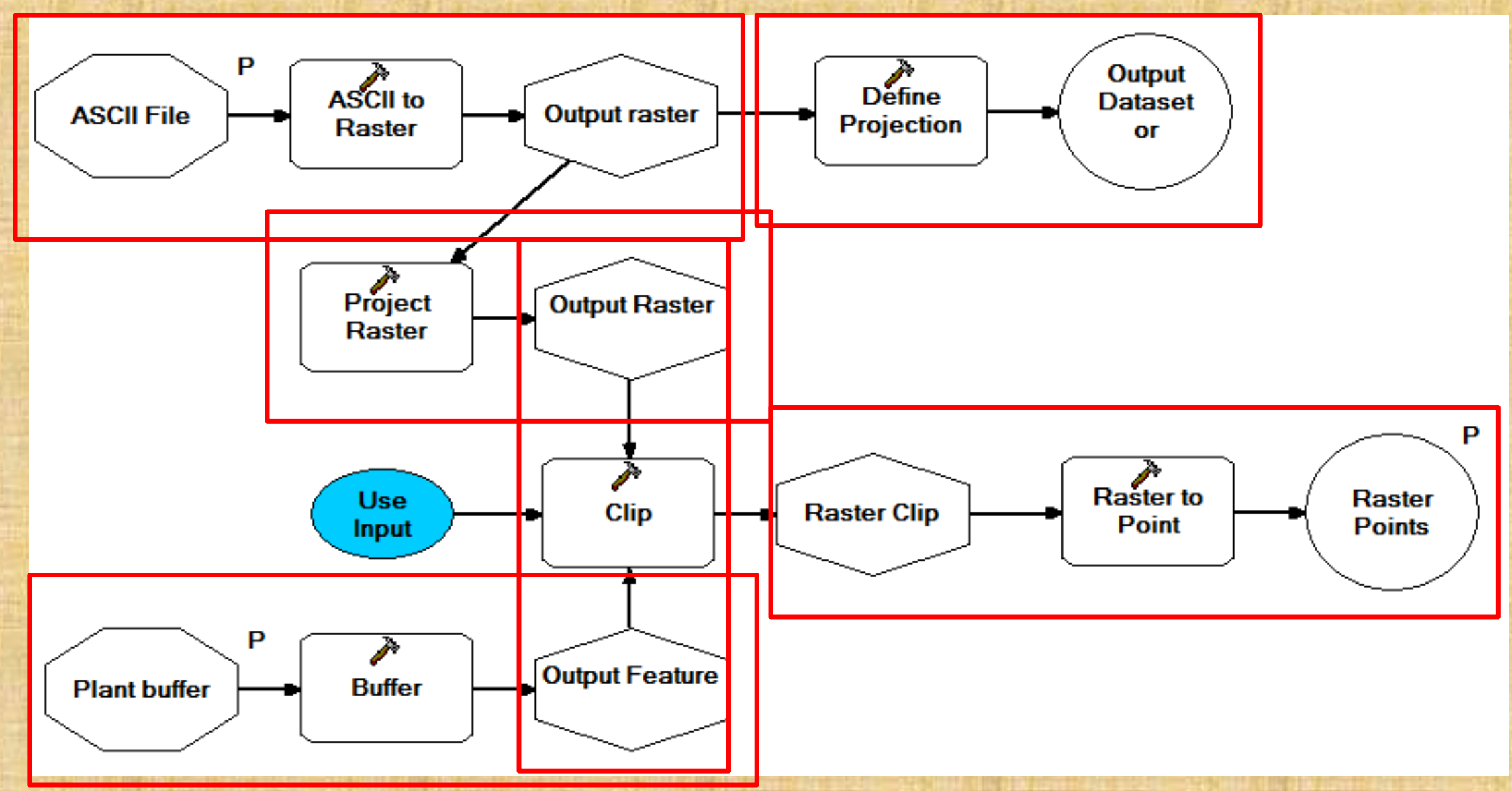




\section{System Design}

Data

\section{Excel Analysis}

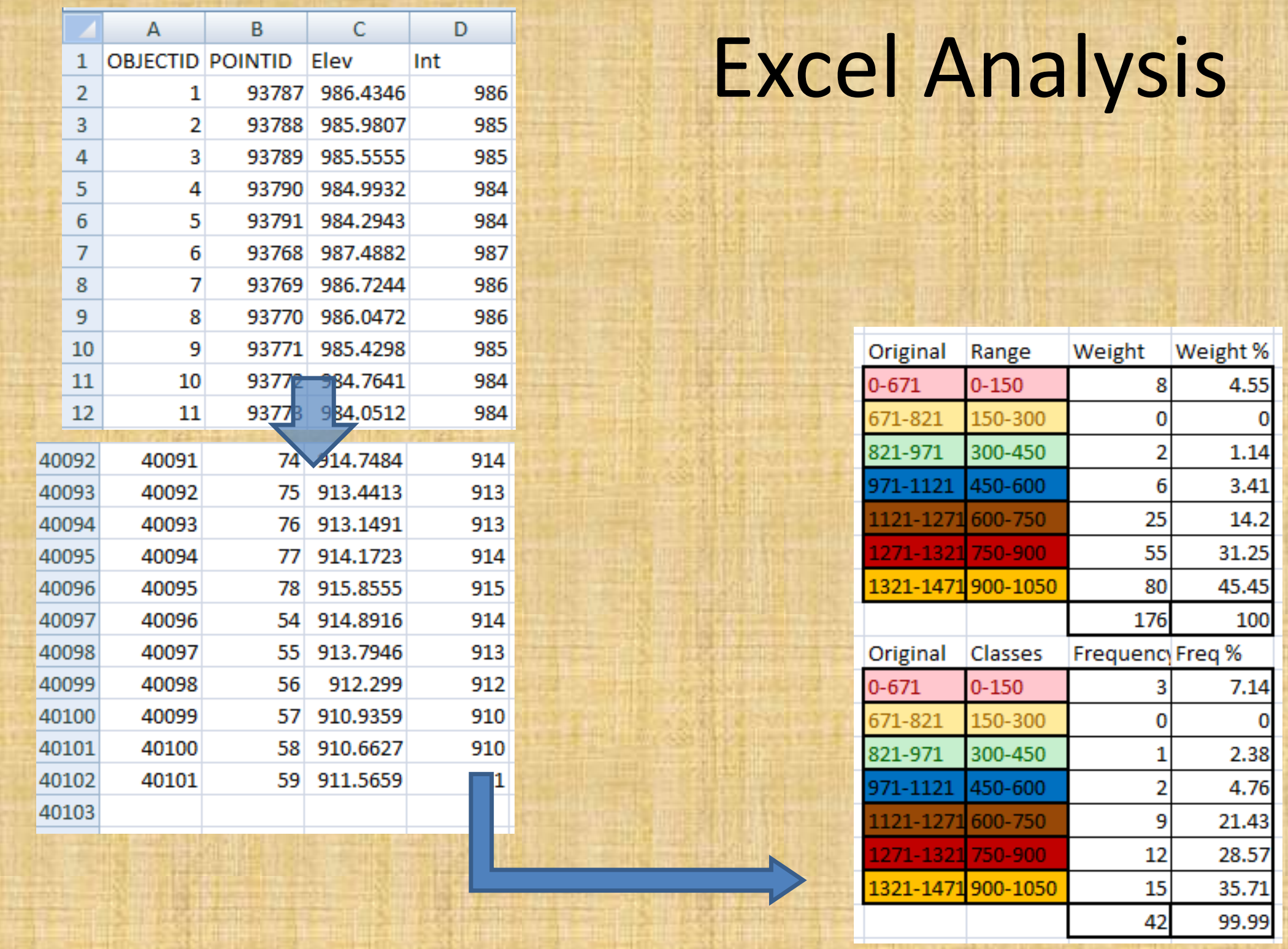




\section{System Design}

\section{Excel Analysis: Aspect}
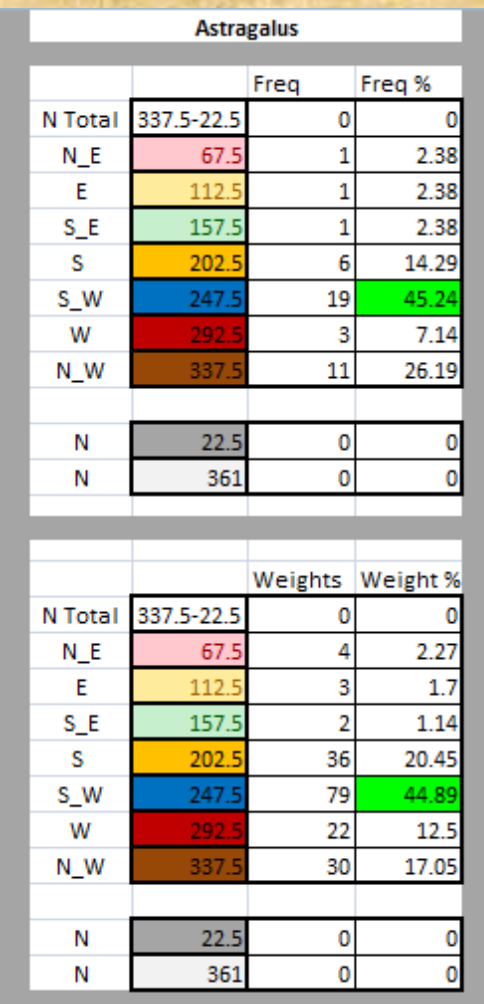
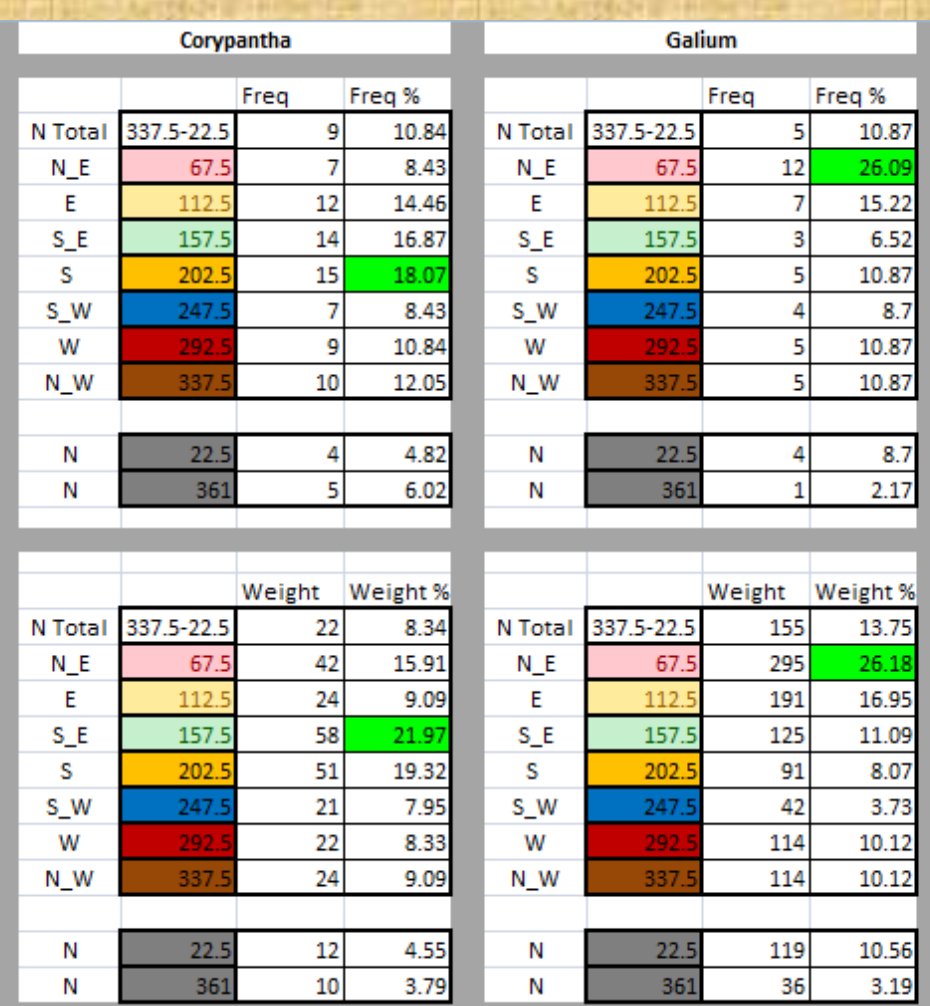

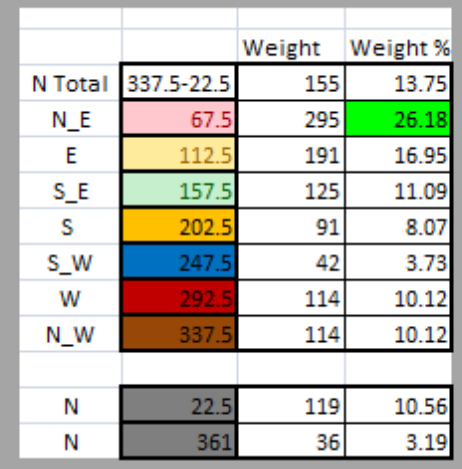

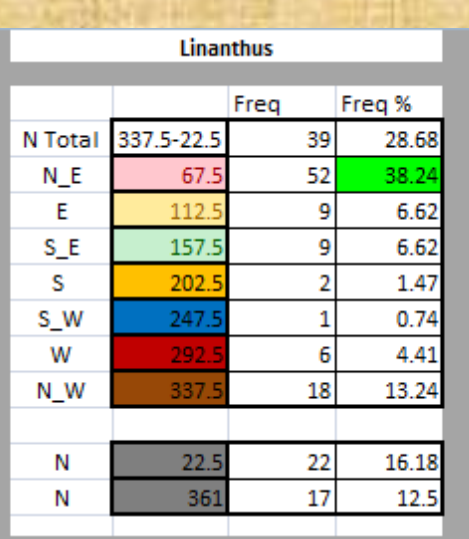
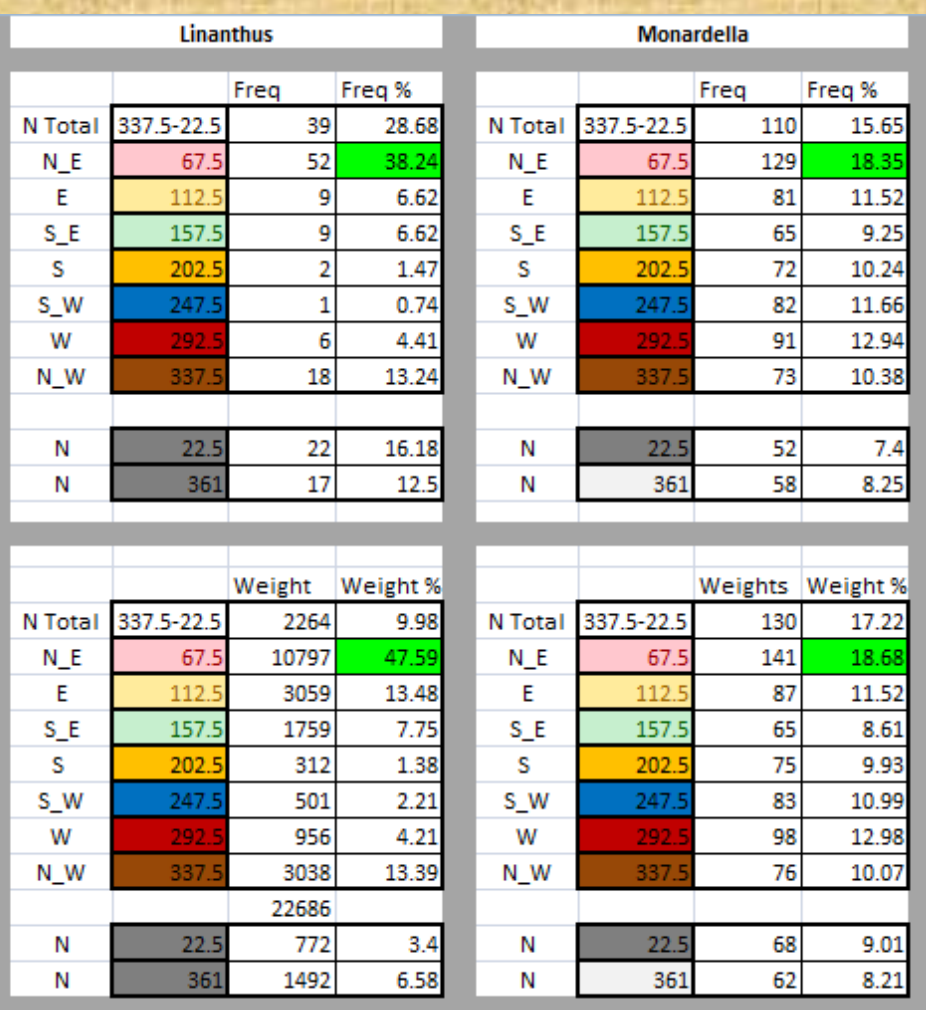

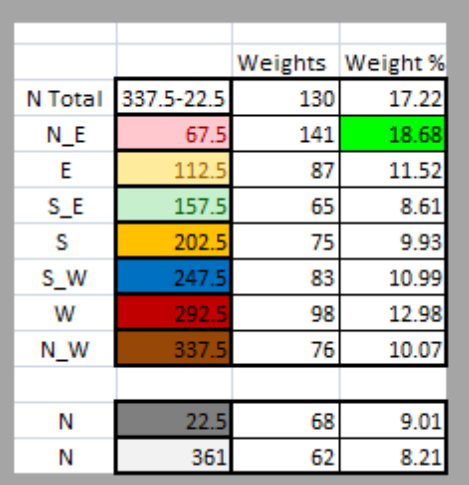




\section{System Design}

\section{Excel Analysis: Slope}

\begin{tabular}{|l|r|r|}
\hline \multicolumn{3}{|c|}{ Astragalus } \\
\hline $0-8$ & 20 & 11.36 \\
\hline $8-16$ & 28 & 15.91 \\
\hline $16-24$ & 34 & 19.32 \\
\hline $24-32$ & 49 & 27.84 \\
\hline $32-40$ & 45 & 25.57 \\
\hline & 176 & 100 \\
\hline
\end{tabular}

\begin{tabular}{|l|r|r|}
\hline \multicolumn{3}{|c|}{ Corypantha } \\
\hline $0-8$ & 205 & 77.65 \\
\hline $8-16$ & 26 & 9.85 \\
\hline $16-24$ & 13 & 4.92 \\
\hline $24-32$ & 16 & 6.06 \\
\hline $32-46$ & 4 & 1.52 \\
\hline & 264 & 100 \\
\hline
\end{tabular}

\begin{tabular}{|l|r|r|}
\hline \multicolumn{3}{|c|}{ Galium } \\
\hline $0-8$ & Weights & Weight \% \\
\hline $8-16$ & 47 & 11.41 \\
\hline $16-24$ & 303 & 73.54 \\
\hline $24-32$ & 42 & 10.19 \\
\hline $32-44$ & 12 & 2.91 \\
\hline & 8 & 1.94 \\
\hline & 412 & 99.99 \\
\hline
\end{tabular}

\begin{tabular}{|l|r|r|}
\hline \multicolumn{3}{|c|}{ Linanthus } \\
\hline $0-8$ & 18045 & 79.54 \\
\hline $8-16$ & 3328 & 14.67 \\
\hline $16-24$ & 1205 & 5.31 \\
\hline $24-32$ & 57 & 0.25 \\
\hline $32-40$ & 51 & 0.22 \\
\hline & 22686 & 99.99 \\
\hline
\end{tabular}

\begin{tabular}{|l|r|r|}
\hline \multicolumn{3}{|c|}{ Monardella } \\
\hline $0-8$ & 349 & 46.23 \\
\hline $8-16$ & 248 & 32.85 \\
\hline $16-24$ & 107 & 14.17 \\
\hline $24-32$ & 44 & 5.83 \\
\hline $32-40$ & 7 & 0.93 \\
\hline & 755 & 100.01 \\
\hline
\end{tabular}

\begin{tabular}{|l|r|r|}
\multicolumn{1}{|c|}{ Freq } & \multicolumn{1}{l|}{ Freq \% } \\
\hline $0-8$ & 6 & 14.28 \\
\hline $8-16$ & 10 & 23.8 \\
\hline $16-24$ & 10 & 23.8 \\
\hline $24-32$ & 7 & 16.66 \\
\hline $32-40$ & 9 & 21.42 \\
\hline & 42 & 99.96 \\
\hline
\end{tabular}

\begin{tabular}{|l|r|r|}
\multicolumn{1}{|c|}{ Freq } & \multicolumn{1}{l|}{ Freq \% } \\
\hline $0-8$ & 56 & 67.47 \\
\hline $8-16$ & 14 & 16.85 \\
\hline $16-24$ & 7 & 8.43 \\
\hline $24-32$ & 4 & 4.81 \\
\hline $32-46$ & 2 & 1.2 \\
\hline & 83 & 98.76 \\
\hline
\end{tabular}

\begin{tabular}{|l|r|r|}
\multicolumn{1}{|l|}{ Freq } & \multicolumn{1}{l|}{ Freq \% } \\
\hline $0-8$ & 13 & 28.26 \\
\hline $8-16$ & 15 & 32.61 \\
\hline $16-24$ & 11 & 23.91 \\
\hline $24-32$ & 5 & 10.86 \\
\hline $32-44$ & 2 & 4.34 \\
\hline & 46 & 99.98 \\
\hline
\end{tabular}

\begin{tabular}{|l|r|r|}
\multicolumn{1}{|c|}{ Freq } & \multicolumn{1}{l|}{ Freq \% } \\
\hline $0-8$ & 100 & 75.53 \\
\hline $8-16$ & 28 & 20.59 \\
\hline $16-24$ & 5 & 3.68 \\
\hline $24-32$ & 2 & 1.48 \\
\hline $32-40$ & 1 & 0.74 \\
\hline & 136 & 102.02 \\
\hline
\end{tabular}

\begin{tabular}{|l|r|r|}
\multicolumn{2}{r|}{ Weights } & Weight \% \\
\hline $0-8$ & 328 & 46.66 \\
\hline $8-16$ & 234 & 33.28 \\
\hline $16-24$ & 96 & 13.65 \\
\hline $24-32$ & 38 & 5.4 \\
\hline $32-40$ & 7 & 0.99 \\
\hline & 703 & 99.98 \\
\hline
\end{tabular}




\section{System Design}

\section{Excel Analysis: DEM}

Astragalus

\begin{tabular}{|l|l|r|r|}
\hline Original & \multicolumn{1}{l}{ Range } & \multicolumn{1}{l}{ Weight } & Weight \% \\
\hline $0-671$ & $0-150$ & 8 & 4.55 \\
\hline $671-821$ & $150-300$ & 0 & 0 \\
\hline $821-971$ & $300-450$ & 2 & 1.14 \\
\hline $971-1121$ & $450-600$ & 6 & 3.41 \\
\hline $1121-1271$ & $600-750$ & 25 & 14.2 \\
\hline $1271-1321$ & $750-900$ & 55 & 31.25 \\
\hline $1321-1471$ & $900-1050$ & 80 & 45.45 \\
\hline
\end{tabular}

\begin{tabular}{|l|l|r|r|}
\hline Original & Classes & Freq & \multicolumn{1}{l|}{ Freq $\%$} \\
\hline $0-671$ & $0-150$ & 3 & 7.14 \\
\hline $671-821$ & $150-300$ & 0 & 0 \\
\hline $821-971$ & $300-450$ & 1 & 2.38 \\
\hline $971-1121$ & $450-600$ & 2 & 4.76 \\
\hline $1121-1271$ & $600-750$ & 9 & 21.43 \\
\hline $1271-1321$ & $750-900$ & 12 & 28.57 \\
\hline $1321-1471$ & $900-1050$ & 15 & 35.71 \\
\hline & & 42 & 99.99 \\
\hline
\end{tabular}

Corypantha

\begin{tabular}{|l|l|r|r|} 
Original & Range & \multicolumn{1}{l}{ Weight } & Weight \% \\
\hline $313-493$ & $0-180$ & 67 & 25.38 \\
\hline $493-673$ & $180-360$ & 27 & 10.23 \\
\hline $673-853$ & $360-540$ & 17 & 6.44 \\
\hline $853-1033$ & $540-720$ & 42 & 15.91 \\
\hline $1033-1213$ & $720-900$ & 28 & 10.61 \\
\hline $1213-1393$ & $900-1080$ & 55 & 20.83 \\
\hline $1393-1513$ & $1080-1200$ & 28 & 10.61 \\
\hline & & 264 & 100.01 \\
\hline
\end{tabular}

\begin{tabular}{|l|l|r|r|}
\hline Original & \multicolumn{1}{l|}{ Range } & Freq & \multicolumn{1}{c|}{ Freq \% } \\
\hline $313-493$ & $0-180$ & 12 & 14.45 \\
\hline $493-673$ & $180-360$ & 7 & 8.43 \\
\hline $673-853$ & $360-540$ & 7 & 8.43 \\
\hline $853-1033$ & $540-720$ & 14 & 16.86 \\
\hline $1033-1213$ & $720-900$ & 17 & 20.48 \\
\hline $1213-1393$ & $900-1080$ & 19 & 22.89 \\
\hline $1393-1513$ & $1080-1200$ & 7 & 8.43 \\
\hline & & 83 & 99.97 \\
\cline { 2 - 4 } & & &
\end{tabular}

Galium

\begin{tabular}{|l|l|r|r|} 
Original & Range & Weight & Weight \% \\
\hline $1012-1072$ & $0-90$ & 11 & 2.67 \\
\hline $1072-1162$ & $90-180$ & 10 & 2.43 \\
\hline $1162-1252$ & $180-270$ & 29 & 7.04 \\
\hline $1252-1342$ & $270-360$ & 282 & 68.45 \\
\hline $1342-1432$ & $360-450$ & 38 & 9.22 \\
\hline $1432-1522$ & $450-540$ & 21 & 5.1 \\
\hline $1522-1642$ & $540-660$ & 21 & 5.1 \\
\hline & & 412 & 100.01 \\
\hline
\end{tabular}

Original
\begin{tabular}{|l|r|r|r|}
\hline $1012-1072$ & $0-90$ & 2 & 4.35 \\
\hline $1072-1162$ & $90-180$ & 3 & 6.51 \\
\hline $1162-1252$ & $180-270$ & 9 & 19.56 \\
\hline $1252-1342$ & $270-360$ & 9 & 19.57 \\
\hline $1342-1432$ & $360-450$ & 10 & 21.74 \\
\hline $1432-1522$ & $450-540$ & 7 & 15.22 \\
\hline $1522-1642$ & $540-660$ & 6 & 13.04 \\
\hline & & 46 & 99.99 \\
\hline
\end{tabular}

Linanthus

\begin{tabular}{|c|c|c|c|}
\hline Original & Range & Weight & Weight $\%$ \\
\hline $833-863$ & $0-45$ & 3837 & 16.91 \\
\hline 863-908 & $45-90$ & 14195 & 62.57 \\
\hline 908-953 & $90-135$ & 3125 & 13.78 \\
\hline 953-998 & $135-180$ & 101 & 0.45 \\
\hline 998-1043 & $180-225$ & 1423 & 6.27 \\
\hline $1043-108$ & $225-270$ & 4 & 0.02 \\
\hline $1088-1103$ & $270-285$ & 1 & \\
\hline & & 22686 & \\
\hline
\end{tabular}

\begin{tabular}{|l|l|r|r|}
\hline Original & Range & Freq & \multicolumn{1}{l|}{ Freq\% } \\
\hline $833-863$ & $0-45$ & 32 & 23.53 \\
\hline $863-908$ & $45-90$ & 60 & 44.11 \\
\hline $908-953$ & $90-135$ & 15 & 11.03 \\
\hline $953-998$ & $135-180$ & 1 & 0.74 \\
\hline $998-1043$ & $180-225$ & 23 & 16.92 \\
\hline $1043-1088$ & $225-270$ & 4 & 2.94 \\
\hline $1088-1103$ & $270-285$ & 1 & 0.74 \\
\hline
\end{tabular}

Monardella

\begin{tabular}{|l|l|r|r|}
\hline Original & Range & Weight & Weight \% \\
\hline $949-999$ & $0-75$ & 31 & 4.11 \\
\hline $999-1074$ & $75-150$ & 43 & 5.7 \\
\hline $1074-114$ & $150-225$ & 69 & 9.14 \\
\hline $1149-1224$ & $225-300$ & 144 & 19.07 \\
\hline $1224-129$ & $300-375$ & 422 & 55.89 \\
\hline $1299-1374$ & $375-450$ & 44 & 5.83 \\
\hline $1374-1644$ & $450-570$ & 2 & 0.26 \\
\hline \multicolumn{2}{|l|}{} & 755 & 100 \\
\hline
\end{tabular}

\begin{tabular}{|c|c|c|c|}
\hline Original & Range & Freq & Freq $\%$ \\
\hline 949-999 & $0-75$ & 19 & 2.7 \\
\hline $999-1074$ & $75-150$ & 43 & 6.11 \\
\hline $1074-1149$ & $150-225$ & 60 & 8.54 \\
\hline $1149-1224$ & $225-300$ & 144 & 20.48 \\
\hline $1224-1299$ & $300-375$ & 396 & 56.32 \\
\hline $1299-1374$ & $375-450$ & 39 & 5.55 \\
\hline $1374-1644$ & $450-570$ & 2 & 0.28 \\
\hline & & 703 & 99.9 \\
\hline
\end{tabular}




\section{Excel Analysis: Temp}

\begin{tabular}{|r|r|r|r|}
\multicolumn{1}{|c|}{} & Frequency & Freq $\%$ \\
\hline $2320-2390$ & 70 & 2 & 4.76 \\
\hline $2320-2460$ & 140 & 7 & 16.67 \\
\hline $2402-2530$ & 210 & 4 & 9.52 \\
\hline $2418-2600$ & 280 & 12 & 28.57 \\
\hline $2419-2670$ & 350 & 3 & 7.14 \\
\hline $2419-2740$ & 420 & 6 & 14.29 \\
\hline $2433-2810$ & 490 & 1 & 2.38 \\
\hline $2436-2880$ & 560 & 5 & 11.9 \\
\hline $2456-2950$ & 630 & 2 & 4.76 \\
\hline & & 42 & 99.99 \\
\hline
\end{tabular}




\section{System Design}

\section{Data}

Implementation

Results

\section{Excel Analysis: Precipitation}

\begin{tabular}{|c|c|c|c|c|c|}
\hline Month & Wet 05 & Month & Wet 09 & Sum: & StdDev: \\
\hline Nov & 0 & Nov & 500 & 190092 & 4133.722 \\
\hline Nov & 0 & Nov & 520 & & \\
\hline Nov & 0 & Nov & 450 & & \\
\hline Nov & 0 & Nov & 466 & & \\
\hline Nov & 9 & Nov & 298 & & \\
\hline Nov & 0 & Nov & 269 & & \\
\hline Dec & 126 & Dec & 5599 & & \\
\hline Dec & 168 & Dec & 5213 & & \\
\hline Dec & 147 & Dec & 4674 & & \\
\hline Dec & 149 & Dec & 4856 & & \\
\hline Dec & 121 & Dec & 2181 & & \\
\hline Dec & 100 & Dec & 3735 & & \\
\hline Jan & 1016 & Jan & 16235 & & \\
\hline Jan & 565 & Jan & 16387 & & \\
\hline Jan & 1150 & Jan & 14210 & & \\
\hline Jan & 805 & Jan & 14971 & & \\
\hline Jan & 813 & Jan & 9845 & & \\
\hline Jan & 494 & Jan & 13093 & & \\
\hline Feb & 2825 & Feb & 4317 & & \\
\hline Feb & 4164 & Feb & 5412 & & \\
\hline Feb & 2590 & Feb & 4010 & & \\
\hline Feb & 3706 & Feb & 4545 & & \\
\hline Feb & 1722 & Feb & 2467 & & \\
\hline Feb & 1895 & Feb & 2770 & & \\
\hline Mar & 4230 & Mar & 272 & & \\
\hline Mar & 4974 & Mar & 1606 & & \\
\hline Mar & 4051 & Mar & 1162 & & \\
\hline Mar & 4833 & Mar & 1631 & & \\
\hline Mar & 1743 & Mar & 2169 & & \\
\hline Mar & 2592 & Mar & 1241 & & \\
\hline
\end{tabular}


System Design

\section{Data}

Near Points

\section{Why?}




\section{System Design}

\section{Results}

\section{Near Points}

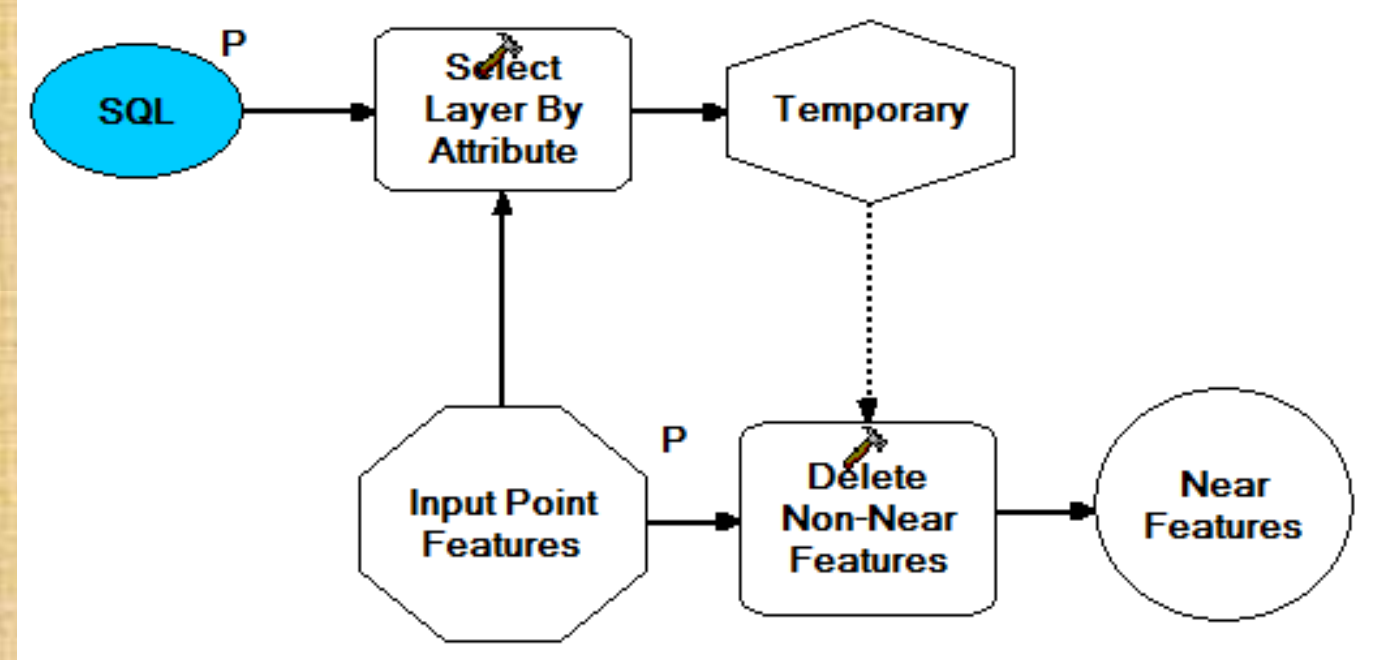

$F I D=28$ OR $\quad F I D=279$ OR FID $=555$ OR FID $=866$ OR $\quad F I D=1175$ OR FID $=1395$ OR $\mathrm{FID}=37 \mathrm{OR} \quad \mathrm{FID}=285 \mathrm{OR}$ FID $=564 \mathrm{OR} F \mathrm{FID}=874 \mathrm{OR} \quad \mathrm{FID}=1192 \mathrm{OR} \mathrm{FID}=1402 \mathrm{OR}$ $\mathrm{FID}=46$ OR $\mathrm{FID}=293 \mathrm{OR} F \mathrm{FID}=573 \mathrm{OR} F \mathrm{FID}=881 \mathrm{OR} \quad \mathrm{FID}=1195 \mathrm{OR} F \mathrm{FID}=1405 \mathrm{OR}$ $\mathrm{FID}=49 \mathrm{OR} \quad \mathrm{FID}=302 \mathrm{OR} \mathrm{FID}=581 \mathrm{OR} F \mathrm{FID}=888 \mathrm{OR} \quad \mathrm{FID}=1198 \mathrm{OR} \mathrm{FID}=1418 \mathrm{OR}$ $F I D=63$ OR $\quad F I D=315$ OR FID $=587$ OR FID $=899$ OR $\quad F I D=1201$ OR FID $=1429$ OR $\mathrm{FID}=70$ OR $\mathrm{FID}=323 \mathrm{OR} \mathrm{FID}=587 \mathrm{OR} \mathrm{FID}=905 \mathrm{OR} \quad \mathrm{FID}=1204 \mathrm{OR} \mathrm{FID}=1433 \mathrm{OR}$ $\mathrm{FID}=76 \mathrm{OR} \quad \mathrm{FID}=323 \mathrm{OR} \mathrm{FID}=588 \mathrm{OR} \quad \mathrm{FID}=914 \mathrm{OR} \quad \mathrm{FID}=1222 \mathrm{OR} \quad \mathrm{FID}=1443 \mathrm{OR}$ $\mathrm{FID}=76$ OR $\quad \mathrm{FID}=334 \mathrm{OR} \quad \mathrm{FID}=597 \mathrm{OR} \quad \mathrm{FID}=922 \mathrm{OR} \quad \mathrm{FID}=1230 \mathrm{OR} \quad \mathrm{FID}=1443 \mathrm{OR}$ $\mathrm{FID}=86 \mathrm{OR} \quad \mathrm{FID}=339 \mathrm{OR} \mathrm{FID}=606 \mathrm{OR} \mathrm{FID}=931 \mathrm{OR} \quad \mathrm{FID}=1234 \mathrm{OR} \mathrm{FID}=1450 \mathrm{OR}$ $\mathrm{FID}=95 \mathrm{OR} \quad \mathrm{FID}=342 \mathrm{OR} \quad \mathrm{FID}=615 \mathrm{OR} \quad \mathrm{FID}=938 \mathrm{OR} \quad \mathrm{FID}=1245 \mathrm{OR} \quad \mathrm{FID}=1458 \mathrm{OR}$ $\mathrm{FID}=103 \mathrm{OR}$ FID $=343 \mathrm{OR} \mathrm{FID}=625 \mathrm{OR} \mathrm{FID}=941 \mathrm{OR} \quad \mathrm{FID}=1253 \mathrm{OR} \mathrm{FID}=1467 \mathrm{OR}$ $\mathrm{FID}=110 \mathrm{OR} F I \mathrm{D}=347 \mathrm{OR} \quad \mathrm{FID}=633 \mathrm{OR} F \mathrm{FID}=957 \mathrm{OR} \quad \mathrm{FID}=1264 \mathrm{OR} \mathrm{FID}=1478 \mathrm{OR}$ $\mathrm{FID}=117 \mathrm{OR}$ FID $=350 \mathrm{OR} \mathrm{FID}=640 \mathrm{OR} \mathrm{FID}=962 \mathrm{OR} \quad \mathrm{FID}=1267 \mathrm{OR} \mathrm{FID}=1481 \mathrm{OR}$ $\mathrm{FID}=128 \mathrm{OR}$ FID $=366 \mathrm{OR} \mathrm{FID}=647 \mathrm{OR} \mathrm{FID}=973 \mathrm{OR} \quad \mathrm{FID}=1273 \mathrm{OR} \mathrm{FID}=1490 \mathrm{OR}$ $\mathrm{FID}=137 \mathrm{OR} \quad \mathrm{FID}=367 \mathrm{OR} \quad \mathrm{FID}=656 \mathrm{OR} \quad \mathrm{FID}=977 \mathrm{OR} \quad \mathrm{FID}=1282 \mathrm{OR} \quad \mathrm{FID}=1497 \mathrm{OR}$ $\mathrm{FID}=140 \mathrm{OR} F \mathrm{FID}=380 \mathrm{OR} \mathrm{FID}=665 \mathrm{OR} \mathrm{FID}=986 \mathrm{OR} \quad \mathrm{FID}=1288 \mathrm{OR} \mathrm{FID}=1506 \mathrm{OR}$ $\mathrm{FID}=144 \mathrm{OR} F \mathrm{FID}=383 \mathrm{OR} \quad \mathrm{FID}=671 \mathrm{OR} \quad \mathrm{FID}=992 \mathrm{OR} \quad \mathrm{FID}=1295 \mathrm{OR} \quad \mathrm{FID}=1515 \mathrm{OR}$ $\mathrm{FID}=147 \mathrm{OR} \mathrm{FID}=386 \mathrm{OR} \mathrm{FID}=678 \mathrm{OR} \mathrm{FID}=1006 \mathrm{OR} \mathrm{FID}=1296 \mathrm{OR} \mathrm{FID}=1522 \mathrm{OR}$ $\mathrm{FID}=150 \mathrm{OR} \quad \mathrm{FID}=394 \mathrm{OR} \mathrm{FID}=688 \mathrm{OR} \mathrm{FID}=1010 \mathrm{OR} \mathrm{FID}=1297 \mathrm{OR} \mathrm{FID}=1531 \mathrm{OR}$ $\mathrm{FID}=154 \mathrm{OR} F \mathrm{FID}=397 \mathrm{OR} \mathrm{FID}=697 \mathrm{OR} \mathrm{FID}=1025 \mathrm{OR} \quad \mathrm{FID}=1298 \mathrm{OR} \mathrm{FID}=1540 \mathrm{OR}$ $F I D=155$ OR FID $=406$ OR FID $=704$ OR FID $=1029$ OR FID $=1301 \mathrm{OR} \quad \mathrm{FID}=1543 \mathrm{OR}$ $\mathrm{FID}=155 \mathrm{OR} \quad \mathrm{FID}=407 \mathrm{OR} \quad \mathrm{FID}=712 \mathrm{OR} \quad \mathrm{FID}=1033 \mathrm{OR} \quad \mathrm{FID}=1303 \mathrm{OR} \quad \mathrm{FID}=1560 \mathrm{OR}$ $\mathrm{FID}=161$ OR FID $=410$ OR FID $=721$ OR FID $=1041$ OR FID $=1307$ OR FID $=1566$ OR $\mathrm{FID}=179 \mathrm{OR}$ FID $=418 \mathrm{OR}$ FID $=732 \mathrm{OR} F \mathrm{FID}=1049 \mathrm{OR}$ FID $=1312 \mathrm{OR}$ FID $=1574 \mathrm{OR}$ $\mathrm{FID}=181 \mathrm{OR}$ FID $=427$ OR FID $=735 \mathrm{OR} \mathrm{FID}=1056 \mathrm{OR} \mathrm{FID}=1313 \mathrm{OR}$ FID $=1582 \mathrm{OR}$ $\mathrm{FID}=184 \mathrm{OR}$ FID $=434 \mathrm{OR} \mathrm{FID}=748 \mathrm{OR} \mathrm{FID}=1066 \mathrm{OR} \mathrm{FID}=1321 \mathrm{OR}$ FID $=1591 \mathrm{OR}$ $\mathrm{FID}=197 \mathrm{OR} \quad \mathrm{FID}=443 \mathrm{OR} \quad \mathrm{FID}=751 \mathrm{OR} \quad \mathrm{FID}=1069 \mathrm{OR} \quad \mathrm{FID}=1324 \mathrm{OR} \mathrm{FID}=1599 \mathrm{OR}$ $\mathrm{FID}=202 \mathrm{OR} F \mathrm{FID}=453 \mathrm{OR} \mathrm{FID}=762 \mathrm{OR} \mathrm{FID}=1080 \mathrm{OR} \mathrm{FID}=1328 \mathrm{OR} \mathrm{FID}=1606 \mathrm{OR}$ $\mathrm{FID}=203 \mathrm{OR} \quad \mathrm{FID}=456 \mathrm{OR} \quad \mathrm{FID}=770 \mathrm{OR} \quad \mathrm{FID}=1089 \mathrm{OR} \quad \mathrm{FID}=1329 \mathrm{OR} \quad \mathrm{FID}=1610 \mathrm{OR}$ $\mathrm{FID}=206$ OR FID $=472$ OR FID $=785$ OR FID $=1097$ OR FID $=1330$ OR FID $=1619$ OR $\mathrm{FID}=207 \mathrm{OR}$ FID $=482$ OR FID $=788$ OR FID $=1108$ OR FID $=1336$ OR FID $=1627 \mathrm{OR}$ $\mathrm{FID}=207 \mathrm{OR} F \mathrm{FID}=486 \mathrm{OR} \mathrm{FID}=797 \mathrm{OR} \mathrm{FID}=1111 \mathrm{OR} \mathrm{FID}=1348 \mathrm{OR} \mathrm{FID}=1635 \mathrm{OR}$ 


\section{System Design}

\section{Weight: NUM_IND}

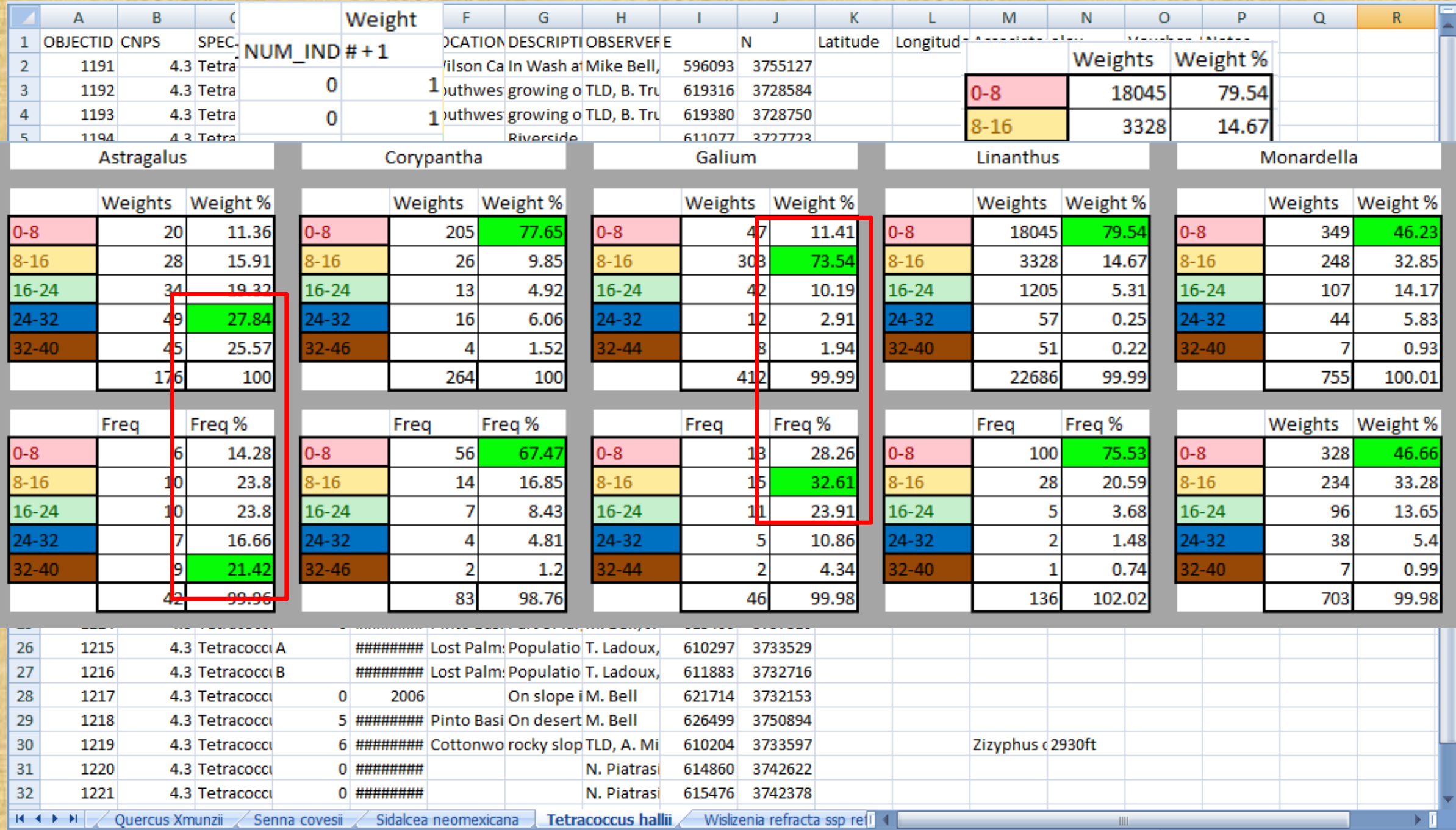




\section{System Design}

\section{Reclassification}

\begin{tabular}{|l|r|r|}
\hline \multicolumn{1}{|c|}{ Freq } & \multicolumn{1}{c|}{ Freq \% } \\
\hline $0-8$ & 56 & 67.47 \\
\hline $8-16$ & 14 & 16.85 \\
\hline $16-24$ & 7 & 8.43 \\
\hline $24-32$ & 4 & 4.81 \\
\hline $32-46$ & 2 & 1.2 \\
\hline & 83 & 98.76 \\
\hline
\end{tabular}

\section{Reclassification}

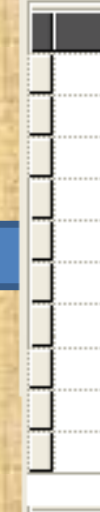

Old values

$-48-949$

949 - 999

999 - 1074

1074 - 1149

$1149-1224$

1224 - 1299

$1299-1374$

1374 - 1644

$1644-3000$

NoData
New values

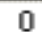

1

3

3

5

9

3

0

0
0

\begin{tabular}{|l|l|l|}
\hline 5 & 7 & 9 \\
\hline $10-20$ & $20-30$ & $>30$ \\
\hline
\end{tabular}

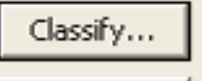

Unique

Add Entry

Delete Entries

Add Entry

Delete Entries

Precision... 


\section{System Design}

\section{Weighted Overlay}

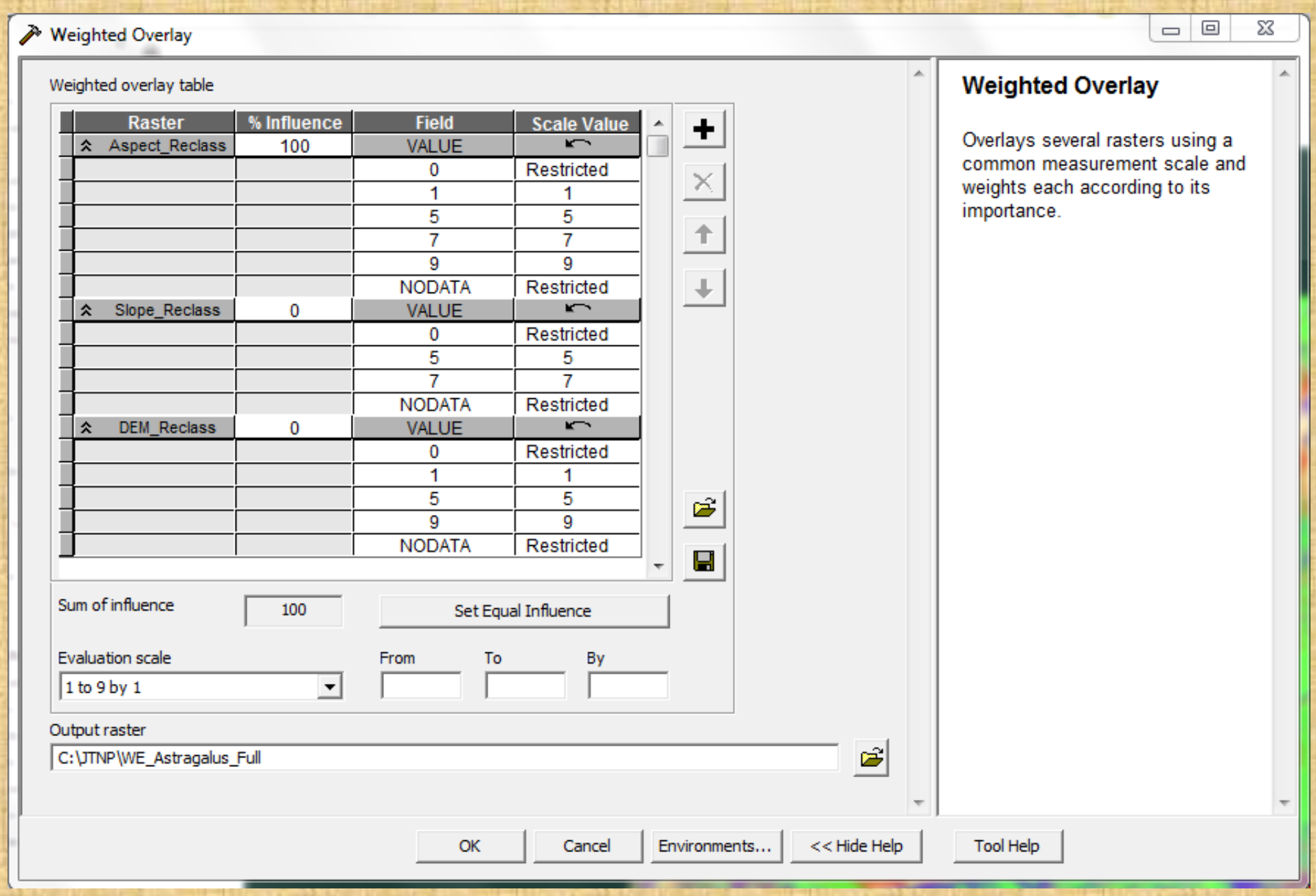




\section{System Design}

\section{Kernel Density}

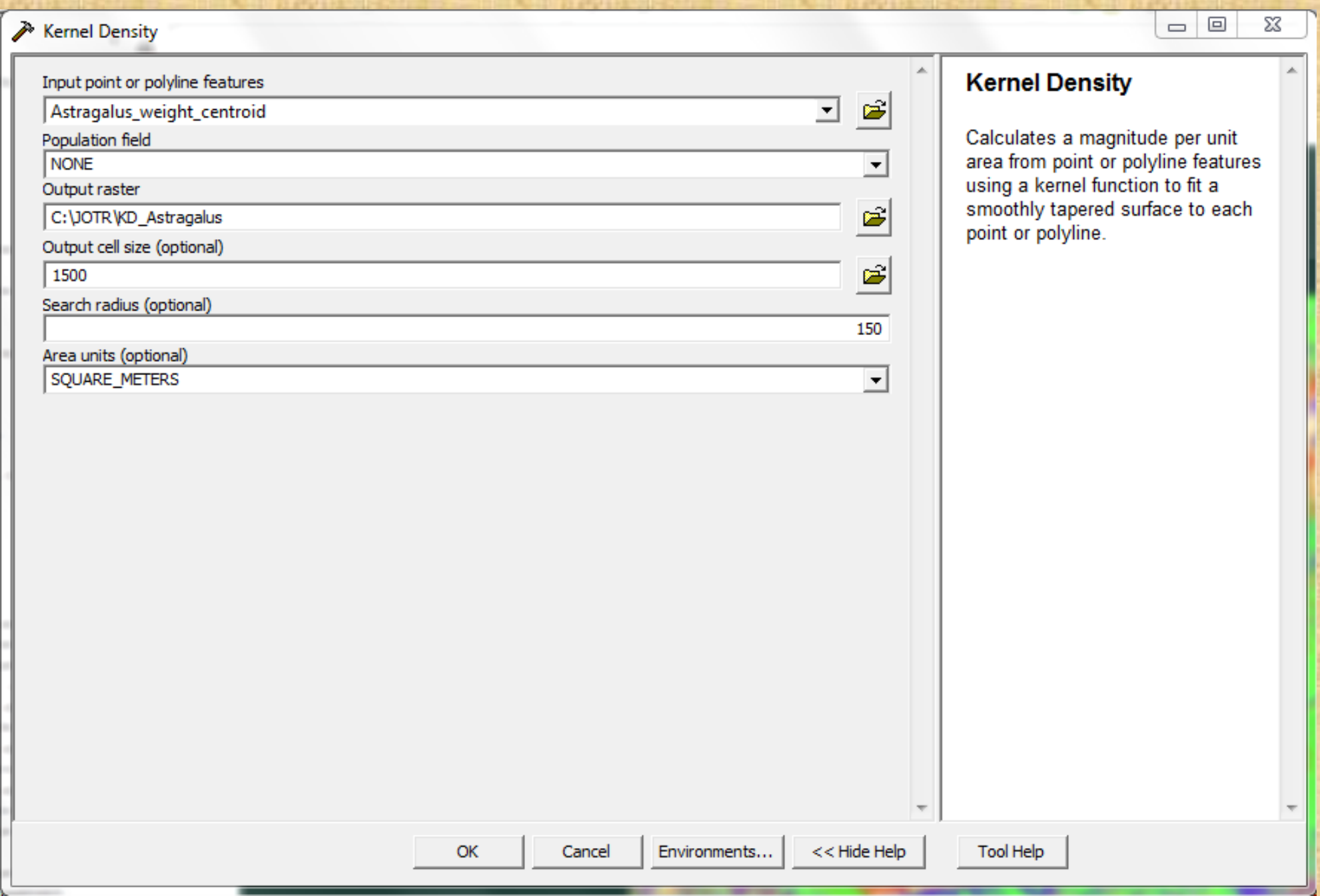




\section{System Design}

\section{Reclassification Analysis}

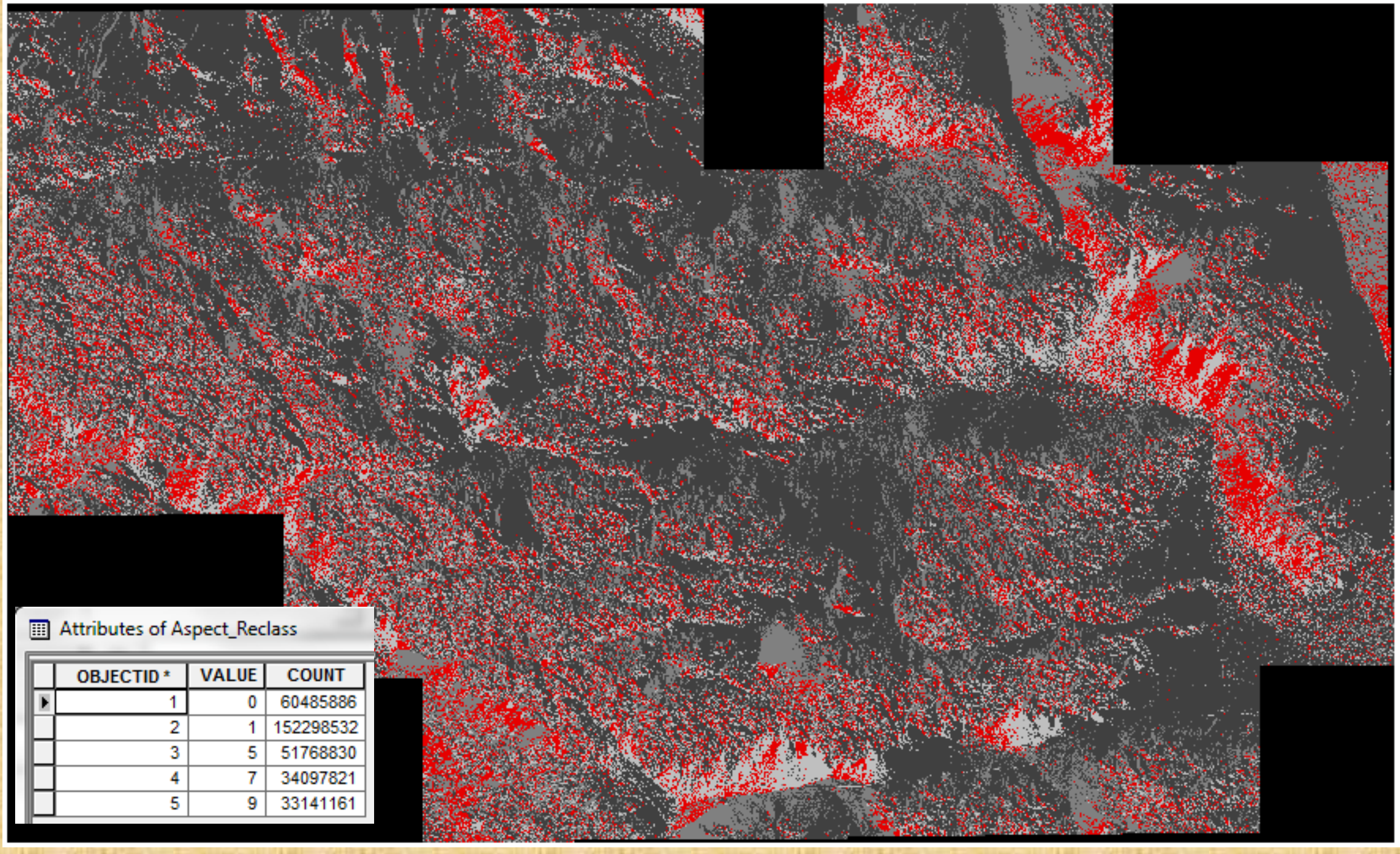




\section{Weighted Overlay}

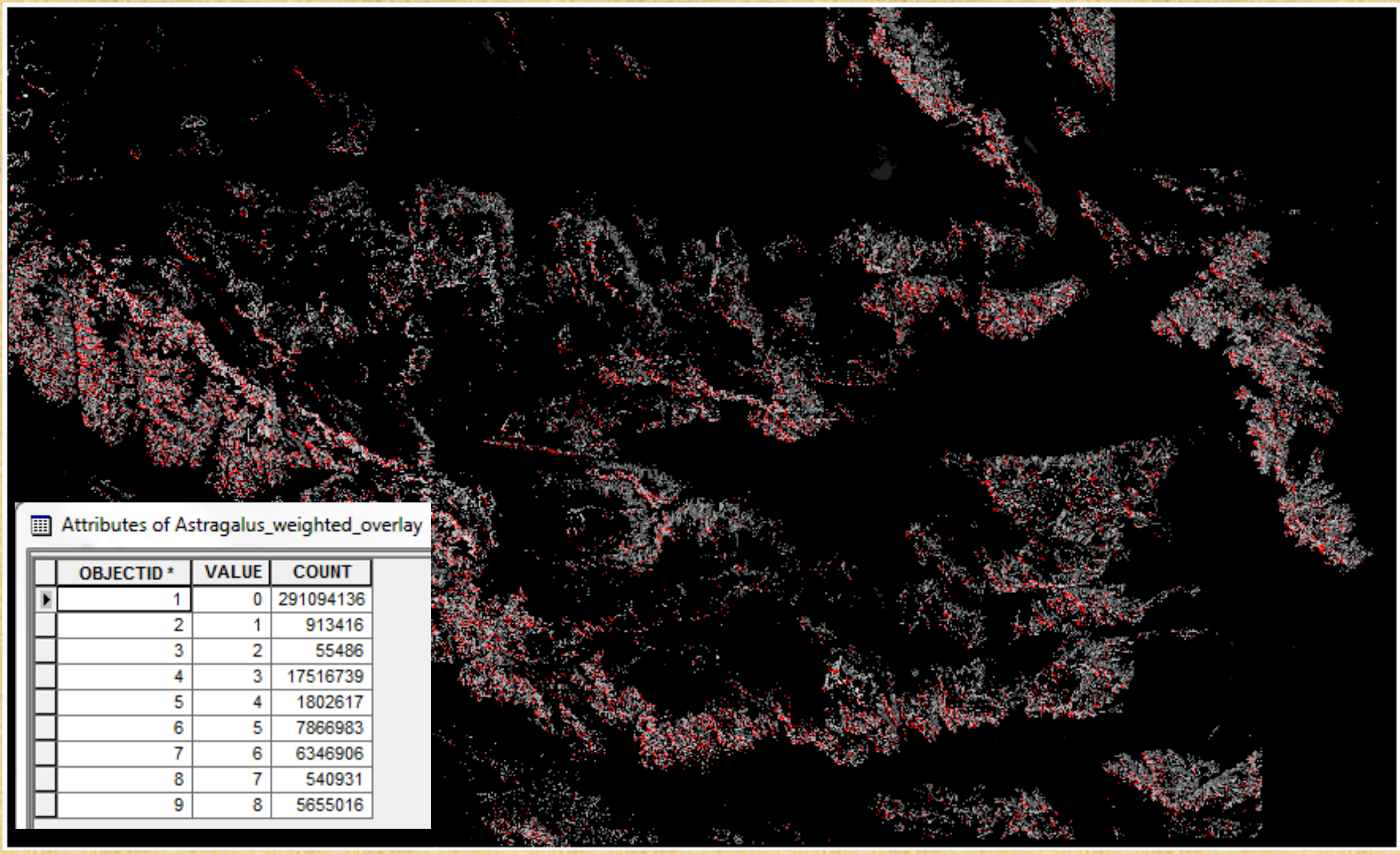




\section{System Design}

\section{Data}

\section{Kernel Density}

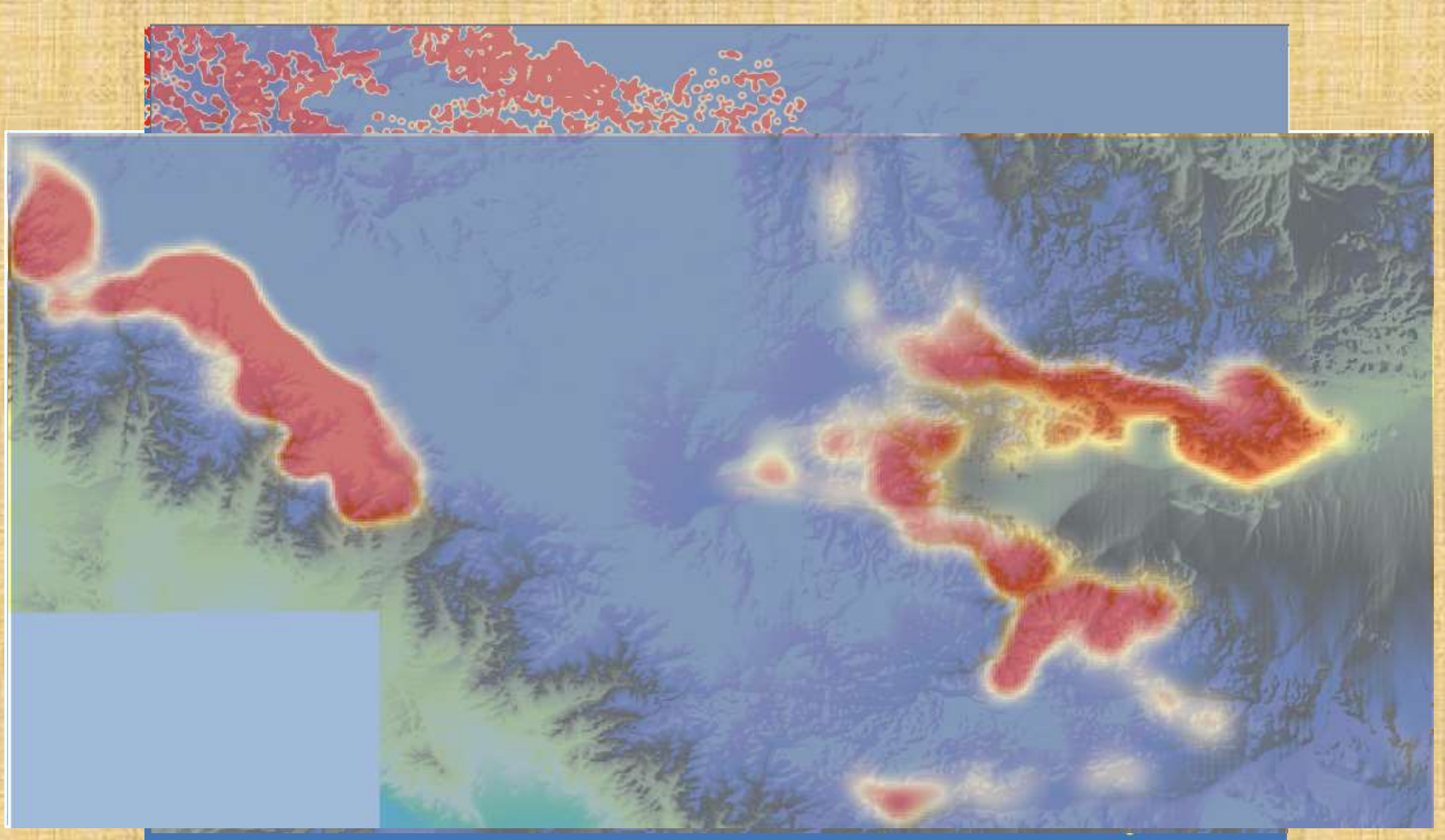




\section{Points versus Weighted Overlay}

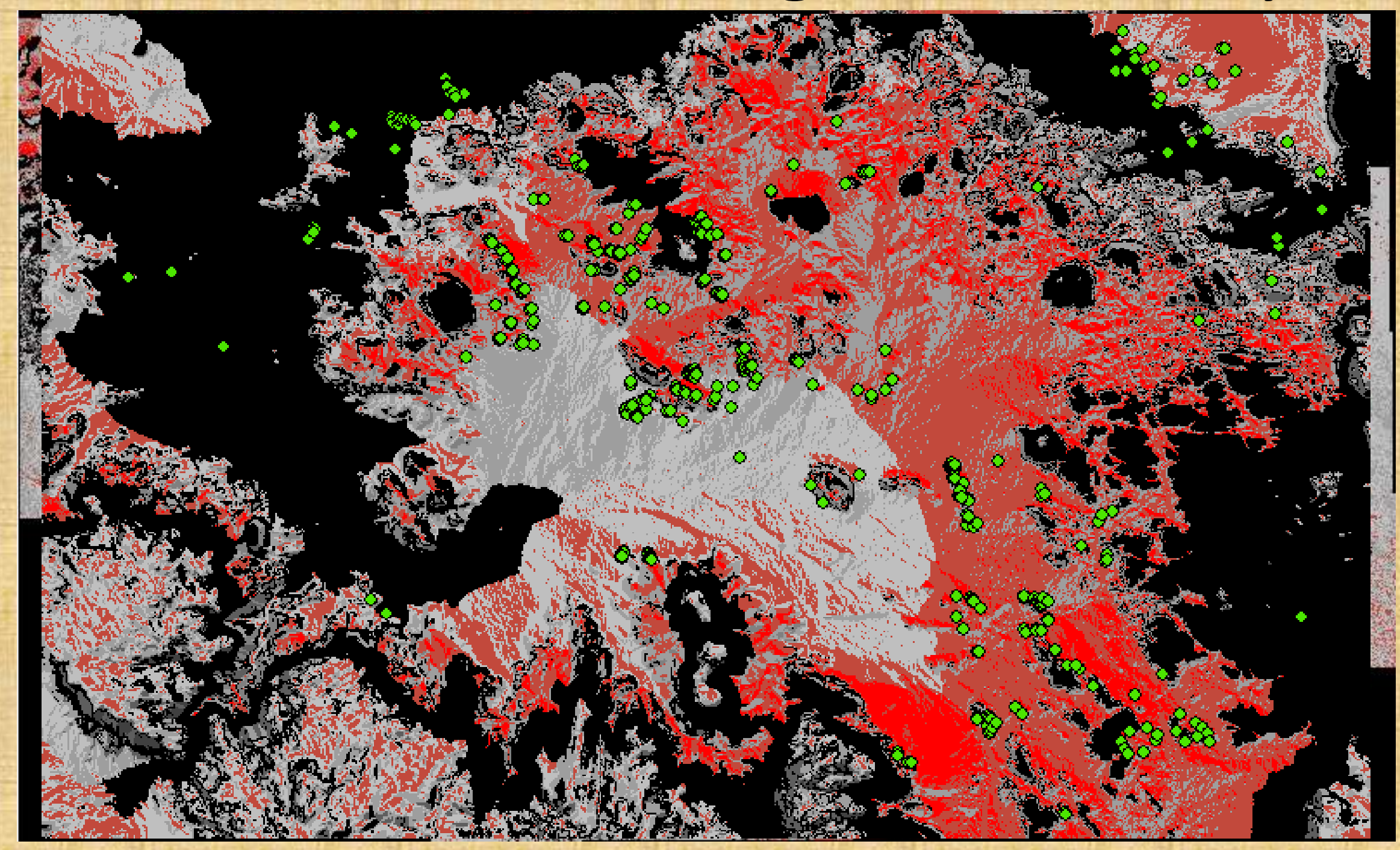




\section{System Design}

\section{Data}

Implementation

\section{Results}

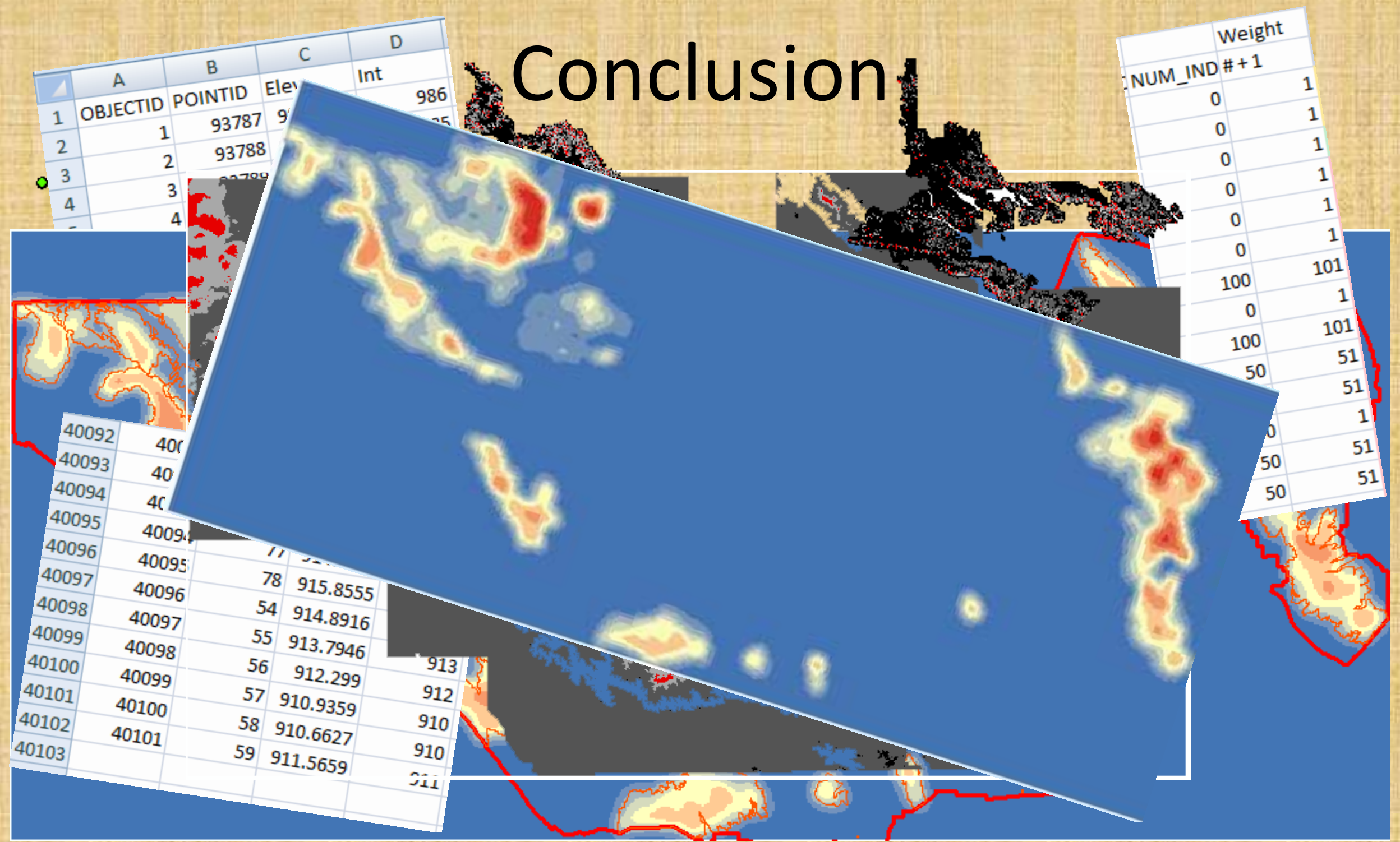




\section{Questions?}
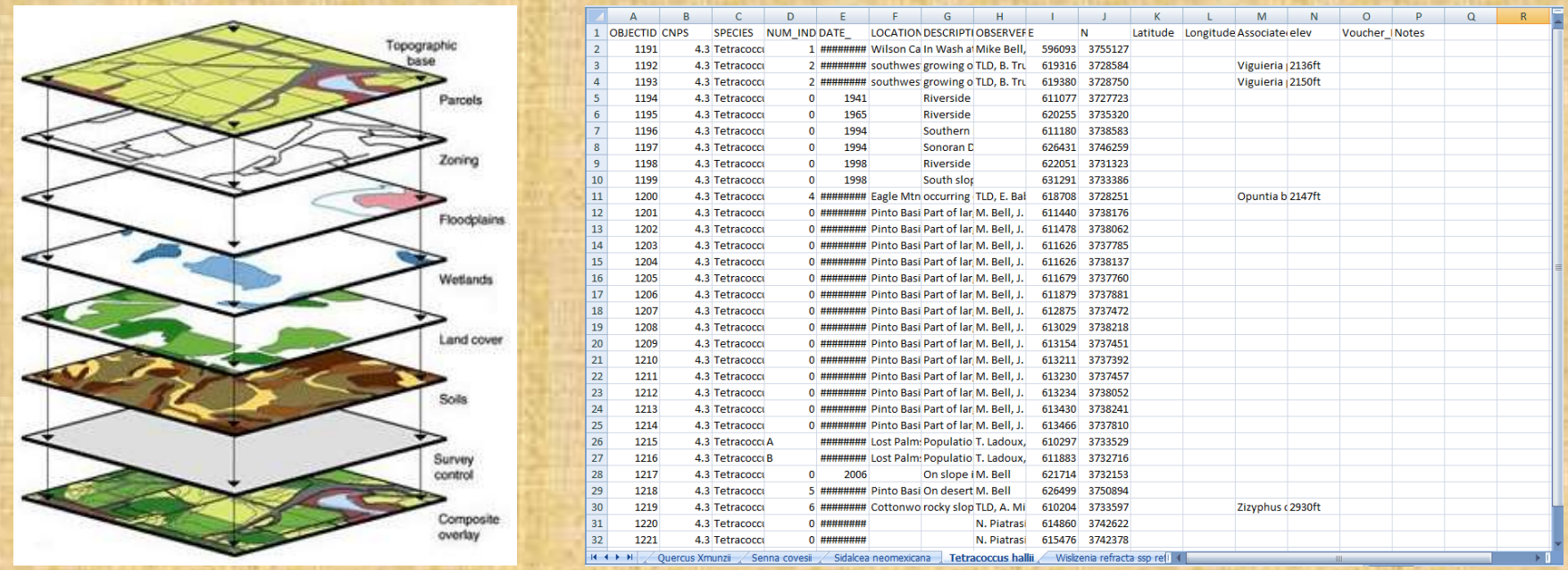

\begin{tabular}{|l|l|l|}
\hline 60 & 24 & 36 \\
\hline 48 & 12 & 6 \\
\hline 6 & 30 & 6 \\
\hline
\end{tabular}

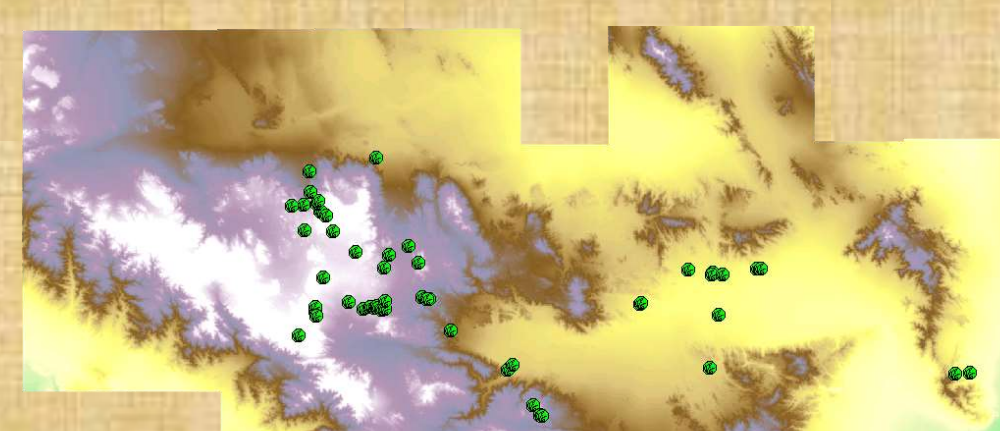

\begin{tabular}{|c|c|c|c|}
\hline Original & Range & Weight & Weight \% \\
\hline $0-671$ & $0-150$ & 8 & 4.55 \\
\hline 671-821 & $150-300$ & 0 & 0 \\
\hline $821-971$ & $300-450$ & 2 & 1.14 \\
\hline 971-1121 & $450-600$ & 6 & 3.41 \\
\hline $1121-1271$ & $600-750$ & 25 & 14.2 \\
\hline $1271-1321$ & $750-900$ & 55 & 31.25 \\
\hline \multirow[t]{2}{*}{ 1321-1471 } & $900-1050$ & 80 & 45.45 \\
\hline & & 176 & 100 \\
\hline Original & Classes & \multicolumn{2}{|c|}{ Frequenç Freq \% } \\
\hline $0-671$ & $0-150$ & 3 & 7.14 \\
\hline 671-821 & $150-300$ & 0 & 0 \\
\hline $821-971$ & $300-450$ & 1 & 2.38 \\
\hline 971-1121 & $450-600$ & 2 & 4.76 \\
\hline $1121-1271$ & $600-750$ & 9 & 21.43 \\
\hline $1271-1321$ & $750-900$ & 12 & 28.57 \\
\hline 1321-1471 & $900-1050$ & 15 & 35.71 \\
\hline & & 42 & 99.99 \\
\hline
\end{tabular}

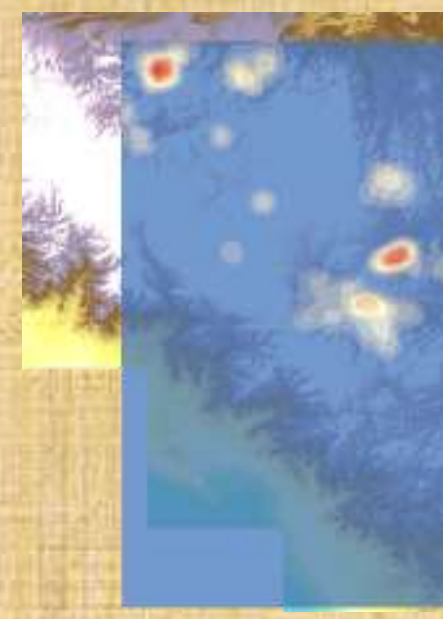

